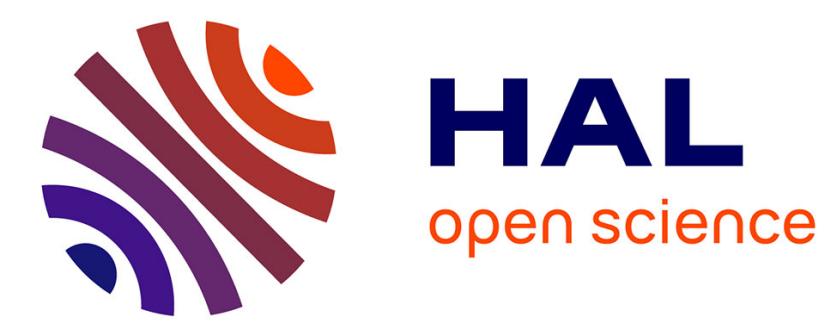

\title{
Gradient Schemes for Stokes problem
}

\author{
Jerome Droniou, Robert Eymard, Pierre Feron
}

\section{To cite this version:}

Jerome Droniou, Robert Eymard, Pierre Feron. Gradient Schemes for Stokes problem. IMA Journal of Numerical Analysis, 2015, 10.1093/imanum/drv061 . hal-01070703v2

\section{HAL Id: hal-01070703 \\ https://hal.science/hal-01070703v2}

Submitted on 17 Mar 2015

HAL is a multi-disciplinary open access archive for the deposit and dissemination of scientific research documents, whether they are published or not. The documents may come from teaching and research institutions in France or abroad, or from public or private research centers.
L'archive ouverte pluridisciplinaire HAL, est destinée au dépôt et à la diffusion de documents scientifiques de niveau recherche, publiés ou non, émanant des établissements d'enseignement et de recherche français ou étrangers, des laboratoires publics ou privés. 


\title{
Gradient Schemes for Stokes problem
}

\author{
Jérôme Droniou* Robert Eymard ${ }^{\dagger}$ and Pierre Feron ${ }^{\ddagger}$
}

March 15, 2015

\begin{abstract}
The gradient scheme framework encompasses several conforming and non-conforming numerical schemes for diffusion equations. We develop here this framework for the approximation of the steady state and transient incompressible Stokes equations with homogeneous Dirichlet boundary conditions. Using this framework, we establish generic convergence results - by error estimates in the case of the steady problem, and by compactness arguments in the case of the transient problem - that are applicable to both old and new schemes for Stokes' equations. Three classical methods (MAC, Taylor-Hood and Crouzeix-Raviart schemes) are shown to fit into the gradient schemes framework; some of the convergence results obtained for those through the framework are new. We also show that a Hybrid Mixed Mimetic scheme, extension of the Crouzeix-Raviart scheme to any polyhedral mesh, can be designed within the gradient scheme framework; this scheme is new for Stokes' equations, and our abstract analysis establishes its convergence along with error estimates.
\end{abstract}

\section{Introduction}

The notion of gradient schemes was designed for linear and nonlinear elliptic and parabolic problems $[12,14,16,21,20]$. Its purpose is to give an abstract unified analysis framework, which provides convergence and error estimates results for a wide variety of numerical methods: finite element methods, nonconforming and mixed finite element methods, hybrid and mixed mimetic finite difference methods, etc. The underlying idea is to write a general discrete weak formulation of the model under which all these methods can be recast; the discrete elements in this weak formulation form the gradient discretisation on which the scheme is built. Then, a few properties are identified on the gradient discretisation, to enable the proof of convergence - along with error estimates in the linear case - of schemes cast in this framework. The work required to prove the convergence of a scheme is then minimal, and solely consist in checking those few properties that are model-independent.

The aim of this paper is to extend this framework to the steady and transient Stokes problems:

$$
\left\{\begin{aligned}
\eta \bar{u}-\Delta \bar{u}+\nabla \bar{p} & =f-\operatorname{div}(G) & & \text { in } \Omega \\
\operatorname{div} \bar{u} & =0 & & \text { in } \Omega \\
\bar{u} & =0 & & \text { on } \partial \Omega
\end{aligned}\right.
$$

where $\bar{u}$ represents the velocity field, $\bar{p}$ is the pressure, the domain $\Omega$ with boundary $\partial \Omega$ is a bounded open set in $\mathbb{R}^{d}, d \geq 1, \eta \in \mathbb{R}^{+}$, and $f$ and $G$ are two given functions defined on $\Omega$, and

$$
\left\{\begin{aligned}
\partial_{t} \bar{u}-\Delta \bar{u}+\nabla \bar{p} & =f-\operatorname{div}(G) & & \text { in } \Omega \times(0, T) \\
\operatorname{div} \bar{u} & =0 & & \text { in } \Omega \times(0, T) \\
\bar{u} & =0 & & \text { on } \partial \Omega \times(0, T) \\
\bar{u}(\cdot, 0) & =\bar{u}_{\text {ini }} & & \text { a.e on } \Omega,
\end{aligned}\right.
$$

*School of Mathematical Sciences, Monash University, Clayton, VIC 3800, Australia. jerome.droniou@monash.edu

${ }^{\dagger}$ LAMA (UMR 8050), UPEM, UPEC, CNRS, F-77454, Marne-la-Vallée, France. Robert.Eymard@u-pem.fr

${ }^{\ddagger}$ LAMA (UMR 8050), UPEM, UPEC, CNRS, F-77454, Marne-la-Vallée, France. Pierre.Feron@u-pem.fr 
where functions $\bar{u}, \bar{p}, f$ and $G$ are now defined on $\Omega \times(0, T)$ with $T>0$, and $\bar{u}_{\text {ini }}$ is a given function defined on $\Omega$.

Different numerical approximations of these models are commonly used in industrial applications. They have been the object of separate analyses. Without exhaustivity, we can cite the following references. In [3], a first error estimate is given for the Taylor-Hood approximation of the Stokes problem, further improved in [32]. In [9], the authors establish an error estimate for the application of the $\mathbb{P}^{1}$-nonconforming method for the velocity, along with piecewise $\mathbb{P}^{0}$ approximation for the pressure, now known as the Crouzeix-Raviart scheme. In the pioneering work [28], the author provides the first convergence proof for the famous Marker-And-Cell (MAC) scheme [24, 29, 33], intensively used in the engineering and fluid numerics frameworks.

All these studies are conducted independently of each other and, even though similar ideas can be found in them, they do not provide a general setting under which schemes for Stokes' equations can be designed and analysed. If someone wants, say, to adapt the Hybrid Mimetic Mixed (HMM) method of [13], which contains the edge-based Mimetic Finite Difference schemes [5], then they would have to (i) design the scheme using ideas from the construction of HMM methods for diffusion equations, and (ii) perform the complete convergence analysis of the scheme for both steady and transient models - probably by drawing ideas from the references previously mentioned. The purpose of this article is to eliminate to an extent this need for different analysis of numerical methods for Stokes' equations. We achieve this result by performing the following, which is the core idea of the gradient schemes framework:

1. We write a generic formulation of numerical approximations of (1) and (2), using a discrete weak formulation of these models based on some choice of discrete spaces and operators;

2. We show that these generic numerical approximations converge to the continuous equations, under a limited number of assumptions the discrete elements involved in the discrete weak formulation;

3. We prove that, for several numerical methods, we can find discrete elements such that the method can be written as in Item 1, and that these discrete elements satisfy the assumptions required in Item 2.

It must be noticed that the work required in Item 3 is much less than the work required to perform the convergence analysis of a given method. Indeed, most of the work already done to establish that these numerical methods are gradient schemes for diffusion equations can be re-used to show that they are gradient schemes for Stokes' equations (the assumptions in Item 2 are model-independent). For example, as seen in the proof of Proposition 4.10, it only takes a few lines to show that the HMM method satisfies all required properties in Item 2, and thus that all our generic convergence results - for steady or transient models - apply to this numerical method.

As we show, the gradient schemes framework also applies to the well-known schemes mentioned above for Stokes' equations (the MAC scheme, the Crouzeix-Raviart scheme and the Taylor-Hood scheme). Convergence results on those schemes have been established in the aforementioned references, but it seems nevertheless that some of the generic convergence results we establish in Item 2 are new even for those methods. For example, for all these well-known schemes the uniform-in-time convergence result under minimal regularity hypotheses (Theorem 3.13) is new to our knowledge. Another example can be found in the error estimate for the steady MAC scheme; the estimate provided in [28] is given in 2D under the regularity hypotheses $\bar{u} \in H^{2}(\Omega)^{d}, \operatorname{curl} \bar{u} \in H^{2}(\Omega)^{d}, p \in H^{2}(\Omega)$; the gradient scheme framework enables us to obtain the order one convergence under the (mostly weaker) assumptions $\bar{u} \in H^{3}(\Omega)^{d}$ and $p \in H^{1}(\Omega)$ (see Remark 4.4). The convergence results for the MAC scheme applied to the transient Stokes' problem also seem totally new.

Finally, another advantage of the generic framework we develop here is that it highlights key features of the numerical methods that lead to robust convergence estimates. For example, [25] exhibits a particular modification of the Crouzeix-Raviart scheme, that has a particular property enabling pressureindependent error estimates. Here, we understand in the gradient scheme framework how to state such 
a property (see (14)) that enables such estimates. Since this is done not for a particular method but in a generic context, this understanding can lead to the development of new robust schemes.

The paper is organised as follows. In Section 2 we detail the discrete space and operators used in gradient discretisations for the Stokes problems, and we write the corresponding gradient schemes. We provide the common properties on the gradient discretisations that are sufficient to establish convergence results for the resulting gradient schemes. These results are stated in Section 3. They cover both the steady and transient problems. For the steady equations, the convergence results are based on error estimates; we first state a basic estimate under the most general hypotheses, and then improved estimates (not depending on the inf-sup constant, or pressure-independent) under more restrictive assumptions on the gradient discretisations. Convergence for the transient model is obtained via compactness techniques, and contains a novel strong $L^{\infty}\left(0, T ; L^{2}(\Omega)^{d}\right)$ convergence result on the velocity without additional regularity assumption required on the data.

Section 4 is devoted to the proof that standard and new schemes fit the gradient scheme framework. The MAC scheme is covered in Section 4.1; note that the extended version of the MAC scheme given in [7] could also be included in the present framework. We show that the gradient discretisation coming from the MAC scheme satisfies the required assumptions, by using discrete functional analysis tools (such as the one developed in [17] for the study of finite volume schemes for elliptic problems). We then consider in Section 4.2 the Taylor-Hood scheme, and the nonconforming Crouzeix-Raviart scheme in Section 4.3. This latter scheme is useful in many industrial applications, due to the approximation of the velocity at the centres of gravity of the faces. This allows for easy couplings with finite volume schemes, when Stokes' equations are coupled with hyperbolic models. In Section 4.4, we present a new scheme for Stokes' equations, which is an extension of the Crouzeix-Raviart scheme to generic polyhedral meshes. This scheme is built from the Hybrid Mixed Mimetic (HMM) methods [13], that include in particular the Mimetic Finite Difference schemes [5], and the SUSHI scheme [18]. It was proved in [14] that HMM schemes for diffusion equations are gradient schemes. Minimal work is required to demonstrate that HMM methods give rise to proper gradient discretisations for the steady and transient Stokes problems; the theorems in Section 3 therefore apply immediately, and give new convergence results for HMM methods on these models.

The proofs of the convergence results stated in Section 3 are provided in Section 5. Several of these proofs are not straightforward, in particular for the transient problem. Appropriate compactness tools have to be designed, which require the definition of suitable discrete norms. A few technical results and proofs are gathered in an appendix (Section 6).

Notations. In the following, if $F$ is a vector space we denote by $\boldsymbol{F}$ the space $F^{d}$. Thus, $\boldsymbol{L}^{2}(\Omega)=L^{2}(\Omega)^{d}$ and $\boldsymbol{H}_{0}^{1}(\Omega)=H_{0}^{1}(\Omega)^{d}$. The space $E(\Omega)$ is the space of fields $v \in \boldsymbol{H}_{0}^{1}(\Omega)$ such that $\operatorname{div} v=0 . L_{0}^{2}(\Omega)$ is the space of functions in $L^{2}(\Omega)$ with a zero mean value over $\Omega$. Finally, $\boldsymbol{H}_{\mathrm{div}}(\Omega)$ is the space of fields $v \in \boldsymbol{L}^{2}(\Omega)$ such that $\operatorname{div} v \in L^{2}(\Omega)$.

\section{Gradient discretisations}

Gradient discretisations provide the foundations, in terms of discrete spaces, operators and properties, upon which the gradient scheme framework is designed.

\subsection{Space}

Definition 2.1 (Gradient discretisation for the steady Stokes problem) Let $\Omega$ be an open bounded domain of $\mathbb{R}^{d}, d \geq 1$. A gradient discretisation $D$ for the incompressible steady Stokes problem, with homogeneous Dirichlet's boundary conditions, is defined by $D=\left(X_{D, 0}, \Pi_{D}, \nabla_{D}, Y_{D}, \chi_{D}, \operatorname{div}_{D}\right)$, where:

1. $X_{D, 0}$ is the finite-dimensional vector space on $\mathbb{R}$ of discrete velocities; 
2. $Y_{D}$ is the finite-dimensional vector space on $\mathbb{R}$ of discrete pressures;

3. The linear mapping $\Pi_{D}: X_{D, 0} \rightarrow \boldsymbol{L}^{2}(\Omega)$ is the reconstruction of the approximate velocity field;

4. The linear mapping $\chi_{D}: Y_{D} \rightarrow L^{2}(\Omega)$ is the reconstruction of the approximate pressure, and must be chosen such that $\left\|\chi_{D} \cdot\right\|_{L^{2}(\Omega)}$ is a norm on $Y_{D}$. We then set $Y_{D, 0}=\left\{q \in Y_{D}: \int_{\Omega} \chi_{D} q \mathrm{~d} x=0\right\}$;

5. The linear mapping $\nabla_{D}: X_{D, 0} \rightarrow \boldsymbol{L}^{2}(\Omega)^{d}$ is the discrete gradient operator. It must be chosen such that $\|\cdot\|_{D}:=\left\|\nabla_{D} \cdot\right\|_{L^{2}(\Omega)^{d}}$ is a norm on $X_{D, 0}$;

6. The linear mapping $\operatorname{div}_{D}: X_{D, 0} \rightarrow L^{2}(\Omega)$ is the discrete divergence operator.

Remark 2.2 (Boundary conditions) Gradient discretisations adapted to other boundary conditions than the homogeneous Dirichlet conditions can be easily designed, see [12].

The coercivity of a sequence of gradient discretisations ensures that a discrete Poincaré inequality, a control of the discrete divergence and a discrete Ladyzenskaja-Babuka-Brezzi (LBB) conditions can be established, all uniform along the sequence of discretisations.

Definition 2.3 (Coercivity) Let $D$ be a discretisation in the sense of Definition 2.1. Let $C_{D}$ and $\beta_{D}$ be defined by

$$
\begin{aligned}
C_{D} & =\max _{v \in X_{D, 0},\|v\|_{D}=1}\left\|\Pi_{D} v\right\|_{L^{2}(\Omega)}+\max _{v \in X_{D, 0},\|v\|_{D}=1}\left\|\operatorname{div}_{D} v\right\|_{L^{2}(\Omega)}, \\
\beta_{D} & =\min \left\{\max _{v \in X_{D, 0},\|v\|_{D}=1} \int_{\Omega} \chi_{D} q \operatorname{div}_{D} v \mathrm{~d} x: q \in Y_{D, 0} \text { such that }\left\|\chi_{D} q\right\|_{L^{2}(\Omega)}=1\right\} .
\end{aligned}
$$

A sequence $\left(D_{m}\right)_{m \in \mathbb{N}}$ of gradient discretisation is coercive if there exist $C_{P} \geq 0$ and $\beta>0$ such that $C_{D_{m}} \leq C_{P}$ and $\beta_{D_{m}} \geq \beta$, for all $m \in \mathbb{N}$.

The consistency of a sequence of gradient discretisations states that the discrete space and operators "fill in" the continuous space as the discretisation is refined.

Definition 2.4 (Consistency) Let $D$ be a gradient discretisation in the sense of Definition 2.1, and let $S_{D}: \boldsymbol{H}_{0}^{1}(\Omega) \rightarrow[0,+\infty)$ and $\widetilde{S}_{D}: L_{0}^{2}(\Omega) \rightarrow[0,+\infty)$ be defined by

$$
\forall \varphi \in \boldsymbol{H}_{0}^{1}(\Omega), \quad S_{D}(\varphi)=\min _{v \in X_{D, 0}}\left(\left\|\Pi_{D} v-\varphi\right\|_{L^{2}(\Omega)}+\left\|\nabla_{D} v-\nabla \varphi\right\|_{L^{2}(\Omega)^{d}}+\left\|\operatorname{div}{ }_{D} v-\operatorname{div} \varphi\right\|_{L^{2}(\Omega)}\right)
$$

and

$$
\forall \psi \in L_{0}^{2}(\Omega), \quad \widetilde{S}_{D}(\psi)=\min _{z \in Y_{D, 0}}\left\|\chi_{D} z-\psi\right\|_{L^{2}(\Omega)} .
$$

A sequence $\left(D_{m}\right)_{m \in \mathbb{N}}$ of gradient discretisation is consistent if, for all $\varphi \in \boldsymbol{H}_{0}^{1}(\Omega), S_{D_{m}}(\varphi)$ tends to 0 as $m \rightarrow \infty$ and, for all $\psi \in L_{0}^{2}(\Omega), \widetilde{S}_{D_{m}}(\psi)$ tends to 0 as $m \rightarrow \infty$.

The discrete differential operators of a gradient discretisation may not satisfy an exact Green-Stokes formula, since the discrete divergence and gradient may not be the continuous ones for non-conforming methods. If the conformity defect (i.e. the amount by which the Green-Stokes formula is not satisfied) of a sequence of gradient discretisations goes to 0 , we say that the sequence is limit-conforming.

Definition 2.5 (Limit-conformity) Let $D$ be a gradient discretisation in the sense of Definition 2.1 and let $\bar{W}_{D}: Z(\Omega) \rightarrow[0,+\infty)$, with $Z(\Omega)=\left\{(\varphi, \psi) \in \boldsymbol{L}^{2}(\Omega)^{d} \times L^{2}(\Omega): \operatorname{div} \varphi-\nabla \psi \in \boldsymbol{L}^{2}(\Omega)\right\}$, be defined by

$$
\forall(\varphi, \psi) \in Z(\Omega), \quad \bar{W}_{D}(\varphi, \psi)=\max _{\substack{v \in X_{D, 0} \\\|v\|_{D}=1}}\left(\int_{\Omega}\left[\nabla_{D} v: \varphi+\Pi_{D} v \cdot(\operatorname{div} \varphi-\nabla \psi)-\psi \operatorname{div}_{D} v\right] \mathrm{d} x\right)
$$


A sequence $\left(D_{m}\right)_{m \in \mathbb{N}}$ of gradient discretisation is limit-conforming if, for all $(\varphi, \psi) \in Z(\Omega), \bar{W}_{D_{m}}(\varphi, \psi)$ tends to 0 as $m \rightarrow \infty$.

The next proposition provides an easy way to check the limit-conformity of a sequence of gradient discretisations in the case where $\Omega$ is locally star-shaped (which is always the case in the cases considered in Section 4, see Remark 4.2). The proof of this proposition is done in an appendix (Section 6.1).

Proposition 2.6 (Characterisation of limit-conformity) We assume that $\Omega$ is a locally star-shaped open bounded domain of $\mathbb{R}^{d}$. If $D$ is a gradient discretisation in the sense of Definition 2.1, we define

$$
\forall \varphi \in \boldsymbol{H}_{\mathrm{div}}(\Omega)^{d}, W_{D}(\varphi)=\max _{v \in X_{D, 0},\|v\|_{D}=1}\left(\int_{\Omega}\left(\nabla_{D} v: \varphi+\Pi_{D} v \cdot \operatorname{div} \varphi\right) \mathrm{d} x\right)
$$

and

$$
\forall \psi \in H^{1}(\Omega), \widetilde{W}_{D}(\psi)=\max _{v \in X_{D, 0},\|v\|_{D}=1}\left(\int_{\Omega}\left(\Pi_{D} v \cdot \nabla \psi+\psi \operatorname{div}_{D} v\right) \mathrm{d} x\right) .
$$

Let $\left(D_{m}\right)_{m \in \mathbb{N}}$ be a coercive sequence of gradient discretisation in the sense of Definition 2.3. Then $\left(D_{m}\right)_{m \in \mathbb{N}}$ is limit-conforming in the sense of Definition 2.5 if and only if $W_{D_{m}}(\varphi) \rightarrow 0$ as $m \rightarrow \infty$ for all $\varphi \in \boldsymbol{H}_{\mathrm{div}}(\Omega)^{d}$, and $\widetilde{W}_{D_{m}}(\psi) \rightarrow 0$ as $m \rightarrow \infty$ and for all $\psi \in H^{1}(\Omega)$.

Remark 2.7 As in [19, 12], the consistency of a coercive sequence of gradient discretisation only needs to be checked on dense subspaces of $\boldsymbol{H}_{0}^{1}(\Omega)$ and $L_{0}^{2}(\Omega)$. This is also true for limit-conformity, see the proof of Proposition 2.6 in the appendix.

\subsection{Space-time}

The notion of gradient discretisation for transient problems requires the addition of time steps and an interpolation (not necessarily linear) of the initial condition.

Definition 2.8 (space-time gradient discretisation) Let $\Omega$ be a bounded open set of $\mathbb{R}^{d}, d \geq 1$, and let $T>0$. A space-time gradient discretisation $D$ for the transient Stokes problem, with homogeneous Dirichlet boundary conditions, is defined by a family $D=\left(X_{D, 0}, \Pi_{D}, \nabla_{D}, Y_{D}, \chi_{D}, \operatorname{div}_{D},\left(t^{(n)}\right)_{n=0, \ldots, N}, J_{D}\right)$ where:

- $D^{s}=\left(X_{D, 0}, \Pi_{D}, \nabla_{D}, Y_{D}, \chi_{D}, \operatorname{div}_{D}\right)$ a gradient discretisation of $\Omega$ in the sense of Definition 2.1;

- $J_{D}: \boldsymbol{L}^{2}(\Omega) \rightarrow X_{D, 0}$ an interpolation operator;

- $t^{(0)}=0<t^{(1)}<\ldots<t^{(N)}=T$.

We define $\delta t^{n+\frac{1}{2}}=t^{(n+1)}-t^{(n)}$ for all $n=0, \ldots, N-1$, and $\delta t_{D}=\max _{n=0, \ldots, N-1}\left(\delta t^{n+\frac{1}{2}}\right)$.

A sequence of space-time gradient discretisation $\left(D_{m}\right)_{m \in \mathbb{N}}$ is coercive (resp. limit-conforming) if its spatial component $\left(D_{m}^{s}\right)_{m \in \mathbb{N}}$ is coercive (resp. limit-conforming). The consistency of a space-time gradient discretisation imposes additional assumptions on the time stepping and the interpolation of the initial conditions.

Definition 2.9 (space-time consistency) A sequence $\left(D_{m}\right)_{m \in \mathbb{N}}$ of space-time gradient discretisations in the sense of Definition 2.8 is consistent if

1. $\left(D_{m}^{s}\right)_{m \in \mathbb{N}}$ is consistent in the sense of Definition 2.4;

2. For all $\varphi \in \boldsymbol{L}^{2}(\Omega), \Pi_{D_{m}} J_{D_{m}} \varphi \rightarrow \varphi$ in $\boldsymbol{L}^{2}(\Omega)$ as $m \rightarrow \infty$;

3. $\delta t_{D_{m}} \rightarrow 0$ as $m \rightarrow \infty$. 


\section{Gradient schemes and main results}

\subsection{Steady Stokes problem}

Our assumptions for the steady Stokes problem (1) are the following:

$$
\begin{aligned}
& \Omega \text { is an open bounded Lipschitz domain of } \mathbb{R}^{d}(d \geq 1), \\
& f \in \boldsymbol{L}^{2}(\Omega), G \in \boldsymbol{L}^{2}(\Omega)^{d} \text { and } \eta \in[0,+\infty) .
\end{aligned}
$$

Definition 3.1 (Weak solution of the steady Stokes problem) Under Hypotheses (6), $(\bar{u}, \bar{p})$ is a weak solution of (1) if

$$
\left\{\begin{array}{l}
\bar{u} \in \boldsymbol{H}_{0}^{1}(\Omega), \bar{p} \in L_{0}^{2}(\Omega), \\
\eta \int_{\Omega} \bar{u} \cdot \bar{v} \mathrm{~d} x+\int_{\Omega} \nabla \bar{u}: \nabla \bar{v} \mathrm{~d} x-\int_{\Omega} \bar{p} \operatorname{div} \bar{v} \mathrm{~d} x=\int_{\Omega}(f \cdot \bar{v}+G: \nabla \bar{v}) \mathrm{d} x, \quad \forall \bar{v} \in \boldsymbol{H}_{0}^{1}(\Omega), \\
\int_{\Omega} q \operatorname{div} \bar{u} \mathrm{~d} x=0, \quad \forall q \in L_{0}^{2}(\Omega),
\end{array}\right.
$$

where "." is the dot product on $\mathbb{R}^{d}$ and, for $\xi=\left(\xi_{i, j}\right)_{i, j=1, \ldots, d} \in \mathbb{R}^{d \times d}$ and $\chi=\left(\chi_{i, j}\right)_{i, j=1, \ldots, d} \in \mathbb{R}^{d \times d}$, $\xi: \chi=\sum_{i, j=1}^{d} \xi_{i, j} \chi_{i, j}$ is the doubly contracted product on $\mathbb{R}^{d \times d}$.

Remark 3.2 Under Hypotheses (6), the existence and uniqueness of a weak solution $(\bar{u}, \bar{p})$ to Problem (1) in the sense of Definition 3.1 follows from [31, Ch.I, Theorem 2.1].

The gradient scheme for the steady Stokes problem is based on a discretisation of the weak formulation (7), in which the continuous spaces and operators are replaced with discrete ones. In (7), we wrote the property "div $\bar{u}=0$ " using test functions to make clearer this parallel between the weak formulation and the gradient scheme. If $D$ is a gradient discretisation in the sense of Definition 2.1, the corresponding gradient scheme is given by:

$$
\left\{\begin{array}{l}
u \in X_{D, 0}, p \in Y_{D, 0}, \\
\eta \int_{\Omega} \Pi_{D} u \cdot \Pi_{D} v \mathrm{~d} x+\int_{\Omega} \nabla_{D} u: \nabla_{D} v \mathrm{~d} x-\int_{\Omega} \chi_{D} p \operatorname{div}_{D} v \mathrm{~d} x \\
=\int_{\Omega}\left(f \cdot \Pi_{D} v+G: \nabla_{D} v\right) \mathrm{d} x, \quad \forall v \in X_{D, 0} \\
\int_{\Omega} \chi_{D} q \operatorname{div}_{D} u \mathrm{~d} x=0, \quad \forall q \in Y_{D, 0} .
\end{array}\right.
$$

Our main result on gradient schemes for steady Stokes problem is the following theorem.

Theorem 3.3 (Error estimates for the steady Stokes problem) Under Hypotheses (6), let $(\bar{u}, \bar{p})$ be the unique solution of the incompressible steady Stokes problem (1) in the sense of Definition 3.1. Let $D$ be a gradient discretisation in the sense of Definition 2.1 such that $\beta_{D}>0$ (see Definition 2.3). Then there exists a unique $\left(u_{D}, p_{D}\right) \in X_{D, 0} \times Y_{D, 0}$ solution of the gradient scheme (8), and there exists $C_{e}>0$, non-decreasing w.r.t. $\eta, C_{D}$ and $\frac{1}{\beta_{D}}$, such that

$$
\begin{array}{r}
\left\|\bar{u}-\Pi_{D} u_{D}\right\|_{L^{2}(\Omega)}+\left\|\nabla \bar{u}-\nabla_{D} u_{D}\right\|_{L^{2}(\Omega)^{d}}+\left\|\bar{p}-\chi_{D} p\right\|_{L^{2}(\Omega)} \\
\quad \leq C_{e}\left(\bar{W}_{D}(\nabla \bar{u}-G, \bar{p})+S_{D}(\bar{u})+\widetilde{S}_{D}(\bar{p})\right) .
\end{array}
$$

Remark 3.4 As a consequence, if $\left(D_{m}\right)_{m \in \mathbb{N}}$ is a coercive, consistent and limit-conforming sequence of gradient discretisations (see Definitions 2.3, 2.4 and 2.5), and if $\left(u_{m}, p_{m}\right)$ are the solutions to the corresponding gradient schemes, then $\lim _{m \rightarrow \infty} \Pi_{D_{m}} u_{m}=\bar{u}$ in $\boldsymbol{L}^{2}(\Omega), \lim _{m \rightarrow \infty} \nabla_{D_{m}} u_{m}=\nabla \bar{u}$ in $\boldsymbol{L}^{2}(\Omega)^{d}$, and $\lim _{m \rightarrow \infty} \chi_{D_{m}} p_{m}=\bar{p}$ in $L^{2}(\Omega)$. 
The constant $C_{e}$ in the preceding estimate may tend to infinity as $\beta_{D}$ tends to zero. For some gradient schemes, we can obtain an estimate on the velocity which is independent on the constant in the inf-sup condition. If $D$ is a gradient discretisation we define the discrete version of the space $E(\Omega)$ by

$$
E_{D}=\left\{v \in X_{D, 0}: \forall q \in Y_{D, 0}, \int_{\Omega} \chi_{D} q \operatorname{div}_{D} v \mathrm{~d} x=0\right\} .
$$

Theorem 3.5 (Error estimates on the velocity without inf-sup constant) Under the assumptions of Theorem 3.3, we suppose that

$$
\begin{gathered}
\forall v \in X_{D, 0}, \quad \text { if } \int_{\Omega} \chi_{D} q \operatorname{div}_{D} v \mathrm{~d} x=0 \text { for all } q \in Y_{D, 0} \text { then } \operatorname{div}_{D} v=0 \text { a.e. in } \Omega \\
\text { (that is to say, } E_{D}=\left\{v \in X_{D, 0}: \operatorname{div}_{D} v=0 \text { a.e. }\right\} \text { ). }
\end{gathered}
$$

Then

$$
\begin{aligned}
\left\|\nabla \bar{u}-\nabla_{D} u_{D}\right\|_{L^{2}(\Omega)^{d}} & \leq\left(\eta C_{D}+2\right) S_{D, E_{D}}(\bar{u})+\bar{W}_{D}(\nabla \bar{u}-G, \bar{p}) \\
\left\|\bar{u}-\Pi_{D} u_{D}\right\|_{L^{2}(\Omega)} & \leq\left[C_{D}\left(\eta C_{D}+1\right)+1\right] S_{D, E_{D}}(\bar{u})+C_{D} \bar{W}_{D}(\nabla \bar{u}-G, \bar{p}),
\end{aligned}
$$

where $S_{D, E_{D}}(\bar{u})=\min _{v \in E_{D}}\left(\left\|\Pi_{D} v-\bar{u}\right\|_{L^{2}(\Omega)}+\left\|\nabla_{D} v-\nabla \bar{u}\right\|_{L^{2}(\Omega)^{d}}\right)$.

Remark 3.6 Most classical schemes for Stokes problem satisfy (11), and have interpolants $E(\Omega) \rightarrow E_{D}$ which ensure that $S_{D, E_{D}}(\bar{u}) \rightarrow 0$ as the mesh size tend to 0 . This is the case of all the schemes presented in Section 4.

Estimates (12) and (13) on the discrete velocity still depend on the continuous pressure $\bar{p}$. This means that even in the case of purely irrotational forces, with the solution of the Stokes equations $(\bar{u}, \bar{p})=(0, \bar{p})$, the pressure terms can lead to errors on the velocity [25]. For some gradient schemes, this dependency on the pressure can be removed.

Theorem 3.7 (Pressure-independent error-estimates on the velocity) Under the assumptions of Theorem 3.3, we suppose that

$$
\forall v \in E_{D}, \forall \psi \in H^{1}(\Omega), \int_{\Omega} \Pi_{D} v \cdot \nabla \psi \mathrm{d} x=0 .
$$

Then, if $\nabla \bar{u}-G \in \boldsymbol{H}_{\mathrm{div}}(\Omega)$ (which amounts to asking that $\bar{p} \in H^{1}(\Omega)$ ), we have the following pressureindependent estimates on the velocity:

$$
\begin{aligned}
\left\|\nabla \bar{u}-\nabla_{D} u_{D}\right\|_{L^{2}(\Omega)^{d}} & \leq\left(\eta C_{D}+2\right) S_{D, E_{D}}(\bar{u})+W_{D}(\nabla \bar{u}-G) \\
\left\|\bar{u}-\Pi_{D} u_{D}\right\|_{L^{2}(\Omega)} & \leq\left[C_{D}\left(\eta C_{D}+1\right)+1\right] S_{D, E_{D}}(\bar{u})+C_{D} W_{D}(\nabla \bar{u}-G),
\end{aligned}
$$

where $W_{D}$ is defined by (5).

Remark 3.8 In case of purely irrotational forces $(f, G)=(\nabla V, 0)$, then the solution to the Stokes problem is $(\bar{u}, \bar{p})=(0, V)$ and Estimates (15) and (16) show that the velocity is exactly approximated. In other words, for such irrotational forces, the discrete velocity provided by the scheme is zero.

Remark 3.9 Assumption (14) is clearly satisfied by some conforming methods (i.e. $X_{D, 0} \subset \boldsymbol{H}_{0}^{1}(\Omega)$, $\operatorname{div}_{D}=\operatorname{div}, \Pi_{D}=\mathrm{Id}, Y_{D, 0} \subset L^{2}(\Omega)$ and $\left.\chi_{D}=\mathrm{Id}\right)$, provided that the divergences of the discrete velocities lie in the space of the discrete pressures. This is in particular the case for the methods in [23, 34, 35], but this is not true for the Taylor-Hood conforming scheme. 
Assumption (14) is however not satisfied in general by non-conforming methods such as the CrouzeixRaviart scheme, when $\Pi_{D}$ is the "classical" reconstruction of function (see Section 4.3). The action in these schemes of purely irrotational forces on the velocity may lead to poor mass conservation. As suggested in [25], a way to satisfy (14) is to use the Raviart-Thomas reconstruction defined in Remark 4.7.

Remark 3.10 For most numerical methods cast in the gradient schemes framework, it is possible to find explicit bounds on $S_{D}, \widetilde{S}_{D}, \bar{W}_{D}, \bar{W}_{D}$ and $\widetilde{W}_{D}$. Estimates (9), (12), (13), (15) and (16) then give explicit convergence rates for these methods. See Remarks 4.4, 4.6, 4.9 and 4.11.

\subsection{Transient Stokes problem}

We consider the transient Stokes problem (2) under the assumptions

$$
\begin{aligned}
& \Omega \text { is an open bounded Lipschitz domain of } \mathbb{R}^{d}(d \geq 1), T>0, \\
& \bar{u}_{\text {ini }} \in \boldsymbol{L}^{2}(\Omega), f \in \boldsymbol{L}^{2}(\Omega \times(0, T)) \text { and } G \in \boldsymbol{L}^{2}(\Omega \times(0, T))^{d} .
\end{aligned}
$$

The solution of (2) is initially understood in the following weak sense, in which the pressure is eliminated by the choice of divergence-free test functions. Existence and uniqueness of this solution is proved in [31, Ch.III, Theorem 1.1].

Definition 3.11 Under Hypothesis (17), $\bar{u}$ is a weak solution of (2) if

$$
\left\{\begin{array}{l}
\bar{u} \in L^{2}(0, T, E(\Omega)) \\
\int_{0}^{T} \int_{\Omega}-\bar{u} \cdot \partial_{t} \varphi \mathrm{d} x \mathrm{~d} t+\int_{\Omega} \bar{u}_{\mathrm{ini}} \cdot \varphi(\cdot, 0) \mathrm{d} x+\int_{0}^{T} \int_{\Omega} \nabla \bar{u}: \nabla \varphi \mathrm{d} x \mathrm{~d} t \\
\quad=\int_{0}^{T} \int_{\Omega}(f \cdot \varphi+G: \nabla \varphi) \mathrm{d} x \mathrm{~d} t, \quad \forall \varphi=\theta w \text { with } \theta \in C_{c}^{\infty}([0, T)) \text { and } w \in E(\Omega) .
\end{array}\right.
$$

It can however be seen (see Section 6.2) that if $u_{\text {ini }} \in E(\Omega)$ and $\bar{u}$ is the solution of (18), then there exists a pressure $\bar{p}$ such that $(\bar{u}, \bar{p})$ is a solution of (2) in the following sense.

Proposition 3.12 Assume Hypotheses (17) and $u_{\mathrm{ini}} \in E(\Omega)$. We let $\bar{u}$ be the solution of (18). Then there exists $\bar{p}$ such that $(\bar{u}, \bar{p})$ satisfies:

$$
\left\{\begin{array}{c}
\bar{u} \in L^{2}(0, T, E(\Omega)) \cap C\left([0, T] ; \boldsymbol{L}^{2}(\Omega)\right), \partial_{t} \bar{u} \in L^{2}\left(0, T, \boldsymbol{H}^{-1}(\Omega)\right), \bar{p} \in L^{2}\left(0, T, L_{0}^{2}(\Omega)\right), \\
\int_{0}^{T} \int_{\Omega}\left\langle\partial_{t} \bar{u}, \varphi\right\rangle \mathrm{d} x \mathrm{~d} t+\int_{0}^{T} \int_{\Omega} \nabla \bar{u}: \nabla \varphi \mathrm{d} x \mathrm{~d} t-\int_{0}^{T} \int_{\Omega} \bar{p} \operatorname{div} \varphi \mathrm{d} x \mathrm{~d} t \\
=\int_{0}^{T} \int_{\Omega}(f \cdot \varphi+G: \nabla \varphi) \mathrm{d} x \mathrm{~d} t, \quad \forall \varphi \in L^{2}\left(0, T ; \boldsymbol{H}_{0}^{1}(\Omega)\right) \\
\bar{u}(\cdot, 0)=\bar{u}_{\text {ini }} \text { a.e on } \Omega
\end{array}\right.
$$

where $\langle\cdot, \cdot\rangle$ denotes the duality pairing between $\boldsymbol{H}^{-1}(\Omega)$ and $\boldsymbol{H}_{0}^{1}(\Omega)$.

Let $D$ be a space-time gradient discretisation in the sense of Definition 2.8. With the notation

$$
\delta_{D}^{n+\frac{1}{2}} u_{D}=\frac{u_{D}^{(n+1)}-u_{D}^{(n)}}{\delta t^{n+\frac{1}{2}}}
$$


the implicit gradient scheme for (2) is based on the following approximation of (19):

$$
\left\{\begin{array}{l}
u_{D}=\left(u_{D}^{(n)}\right)_{n=0, \ldots, N}, p_{D}=\left(p_{D}^{(n)}\right)_{n=1, \ldots, N} \text { s.t. } u_{D}^{(0)}=J_{D} u_{\text {ini }} \text { and }, \text { for all } n=0, \ldots, N-1, \\
u_{D}^{(n+1)} \in X_{D, 0}, p_{D}^{(n+1)} \in Y_{D, 0}, \\
\int_{\Omega} \Pi_{D} \delta_{D}^{n+\frac{1}{2}} u_{D} \cdot \Pi_{D} v \mathrm{~d} x+\int_{\Omega} \nabla_{D} u_{D}^{(n+1)}: \nabla_{D} v \mathrm{~d} x-\int_{\Omega} \chi_{D} p_{D}^{(n+1)} \operatorname{div}_{D} v \mathrm{~d} x \\
\quad=\frac{1}{\delta t^{n+\frac{1}{2}}} \int_{t^{(n)}}^{t^{(n+1)}} \int_{\Omega}\left(f \cdot \Pi_{D} v+G: \nabla_{D} v\right) \mathrm{d} x \mathrm{~d} t, \quad \forall v \in X_{D, 0} \\
\int_{\Omega} \operatorname{div}_{D} u_{D}^{(n+1)} \chi_{D} q \mathrm{~d} x=0, \quad \forall q \in Y_{D, 0} .
\end{array}\right.
$$

It is common to use $\Pi_{D}$ and $\nabla_{D}$ to denote space-time functions the following way. If $v=\left(v^{n}\right)_{n=0, \ldots, N} \in$ $X_{D, 0}$, the functions $\Pi_{D} v: \Omega \times[0, T] \rightarrow \mathbb{R}^{d}, \nabla_{D} v: \Omega \times(0, T) \rightarrow \mathbb{R}^{d \times d}$ and $\delta_{D} v:(0, T) \rightarrow X_{D, 0}$ are defined by

$$
\begin{aligned}
& \Pi_{D} v(\cdot, 0)=\Pi_{D} v^{0} \text { and } \forall n=0, \ldots, N-1, \forall t \in\left(t^{(n)}, t^{(n+1)}\right], \forall x \in \Omega, \\
& \Pi_{D} v(x, t)=\Pi_{D} v^{(n+1)}(x), \nabla_{D} v(x, t)=\nabla_{D} v^{(n+1)}(x), \text { and } \delta_{D} v(t)=\delta_{D}^{n+\frac{1}{2}} v .
\end{aligned}
$$

Our first convergence result deals only with the velocity.

Theorem 3.13 (Convergence of the velocity for the transient Stokes problem)

Under Hypotheses (17), let $\bar{u}$ be the unique weak solution of the incompressible transient Stokes problem (2) in the sense of Definition 3.11. We take $\left(D_{m}\right)_{m \in \mathbb{N}}$ a sequence of space-time gradient discretisations in the sense of Definition 2.8, that is space-time consistent, limit-conforming and coercive in the sense of Definitions 2.9, 2.5 and 2.3. Then for any $m$ there is a unique solution $\left(u_{D_{m}}, p_{D_{m}}\right)$ to (20) with $D=D_{m}$ and, as $m \rightarrow \infty$,

- $\Pi_{D_{m}} u_{D_{m}}$ converges to $\bar{u}$ in $L^{\infty}\left(0, T ; \boldsymbol{L}^{2}(\Omega)\right)$,

- $\nabla_{D_{m}} u_{D_{m}}$ converges to $\nabla \bar{u}$ in $\boldsymbol{L}^{2}(\Omega \times(0, T))^{d}$.

Remark 3.14 Since the functions $\left(\Pi_{D_{m}} u_{D_{m}}\right)_{m \in \mathbb{N}}$ are piecewise constant in time, their convergence in $L^{\infty}\left(0, T ; \boldsymbol{L}^{2}(\Omega)\right)$ is actually a uniform-in-time convergence (not "uniform a.e. in time").

Under slightly more restrictive (but usual) conditions on the initial data, we can also prove a convergence result on the pressure.

Theorem 3.15 (Convergence of the pressure for the transient Stokes problem) Under the assumptions and notations of Theorem 3.13, we suppose that $G=0, u_{\mathrm{ini}} \in E(\Omega)$, $\left(\left\|J_{D_{m}} u_{\mathrm{ini}}\right\|_{D_{m}}\right)_{m \in \mathbb{N}}$ is bounded and, for all $m \in \mathbb{N}, J_{D_{m}} u_{\mathrm{ini}} \in E_{D_{m}}$ (where $E_{D_{m}}$ is defined by (10) with $D=D_{m}^{s}$, the spatial gradient discretisation corresponding to $D_{m}$ ). Then, as $m \rightarrow \infty$,

- $\Pi_{D_{m}} u_{D_{m}}$ converges to $\bar{u}$ in $L^{\infty}\left(0, T ; \boldsymbol{L}^{2}(\Omega)\right)$,

- $\nabla_{D_{m}} u_{D_{m}}$ converges to $\nabla \bar{u}$ in $\boldsymbol{L}^{2}(\Omega \times(0, T))^{d}$,

- $\chi_{D_{m}} p_{D_{m}}$ weakly converges to $\bar{p}$ in $L^{2}(\Omega \times(0, T))$,

where $(\bar{u}, \bar{p})$ is the weak solution of (2) in the sense (19). 


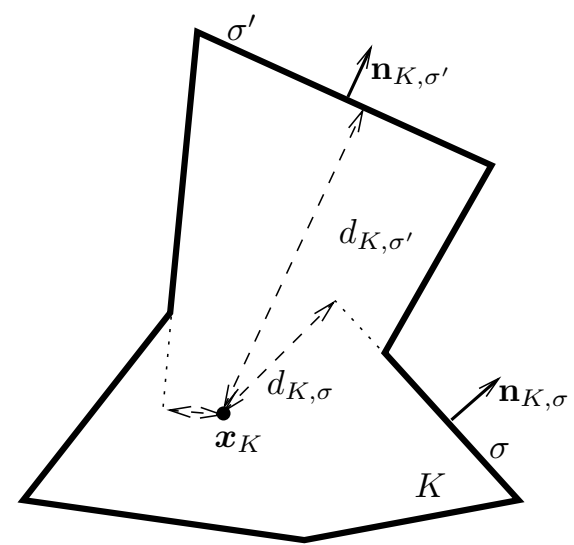

Figure 1: A cell $K$ in a polyhedral mesh

\section{Examples of gradient discretisations}

Although gradient schemes are not necessarily based on meshes, most numerical methods for Stokes' equations are mesh-based. We give here the generic definition of a polyhedral mesh, following [14, 12]. We refer to Figure 1 for some notations.

Definition 4.1 (Polyhedral mesh of $\Omega$ ) Let $\Omega$ be a polyhedral open bounded domain of $\mathbb{R}^{d}, d \geq 1$. A polyhedral mesh of $\Omega$ is a triplet $(\mathcal{M}, \mathcal{E}, \mathcal{P})$ where:

1. $\mathcal{M}$ is a finite family of non empty connected open disjoint subsets of $\Omega$ (the "cells") such that $\bar{\Omega}=\cup_{K \in \mathcal{M}} \bar{K}$. For $K \in \mathcal{M}$ we denote by $\partial K=\bar{K} \backslash K$ the boundary of $K$, by $|K|>0$ the measure of $K$, and by $h_{K}$ the diameter of $K$.

2. $\mathcal{E}=\mathcal{E}_{\text {int }} \cup \mathcal{E}_{\text {ext }}$ is a finite family of disjoint subsets of $\bar{\Omega}$ (the "edges" of the mesh - "faces" in $3 D$ ) such that any $\sigma \in \mathcal{E}$ is a non-empty open subset of a hyperplane of $\mathbb{R}^{d}$. $\mathcal{E}_{\text {int }}$ is the set of edges included in $\Omega$, and $\mathcal{E}_{\text {ext }}$ is the set of edges included in $\partial \Omega$. The $(d-1)$-dimensional measure and the centre of gravity of $\sigma \in \mathcal{E}$ are respectively denoted by $|\sigma|$ and $\bar{x}_{\sigma}$.

We assume that, for all $K \in \mathcal{M}$, there exists a subset $\mathcal{E}_{K}$ of $\mathcal{E}$ such that $\partial K=\cup_{\sigma \in \mathcal{E}_{K}} \bar{\sigma}$. We then set $\mathcal{M}_{\sigma}=\left\{K \in \mathcal{M}: \sigma \in \mathcal{E}_{K}\right\}$ and we assume that, for all $\sigma \in \mathcal{E}$, either $\mathcal{M}_{\sigma}$ has exactly one element and $\sigma \in \mathcal{E}_{\text {ext }}$, or $\mathcal{M}_{\sigma}$ has exactly two elements and $\sigma \in \mathcal{E}_{\text {int }}$. For all $K \in \mathcal{M}$ and $\sigma \in \mathcal{E}_{K}$, we denote by $\mathbf{n}_{K, \sigma}$ the unit vector normal to $\sigma$ outward to $K$.

3. $\mathcal{P}=\left(x_{K}\right)_{K \in \mathcal{M}}$ is a family of points of $\Omega$ indexed by $\mathcal{M}$ such that, for all $K \in \mathcal{M}, K$ is strictly star-shaped with respect to $x_{K}$, meaning that for all $x \in K$ the segment $\left[x_{K}, x\right]$ is included in $K$. We let $d_{K, \sigma}$ be the signed distance between $x_{K}$ and $\sigma$, that is:

$$
d_{K, \sigma}=\left(x-x_{K}\right) \cdot \mathbf{n}_{K, \sigma}, \quad x \in \sigma .
$$

Note that $\left(x-x_{K}\right) \cdot \mathbf{n}_{K, \sigma}$ is constant for $x \in \sigma$, and that it is strictly positive due to $K$ being star-shaped with respect to $x_{K}$.

The size of the polyhedral mesh is defined by $h_{\mathcal{M}}=\max \left\{h_{K}: K \in \mathcal{M}\right\}$.

Remark 4.2 If $\Omega$ has a polyhedral mesh, then $\Omega$ is polyhedral and locally star-shaped. Otherwise, $\Omega$ would have a slit and there would exist edges in the polyhedral discretisation that simultaneously lie on $\partial \Omega$ and have two neighbouring cells, which is prevented by Item 2. 


\subsection{The MAC scheme}

The Marker-And-Cell (MAC) scheme [24, 29, 33] can be easily defined on domains where the boundary is composed of subparts parallel to the axes. Let us show that its basic version fits the gradient scheme framework. For simplicity of presentation, we restrict the presentation to $2 \mathrm{D}$ domains and we further assume that $\Omega=(a, b) \times(c, d)$.

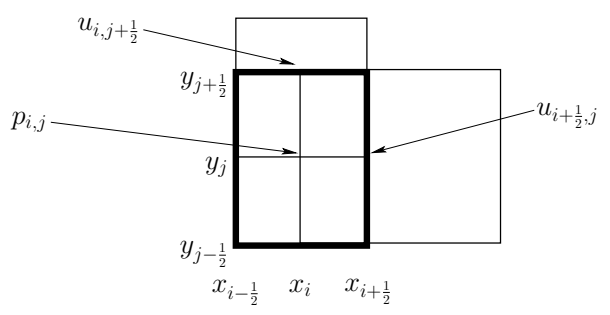

Figure 2: Mesh for the MAC method.

We introduce, for given $N, M \in \mathbb{N}^{\star}$, finite real sequences $x_{\frac{1}{2}}=a<x_{1+\frac{1}{2}} \ldots<b=x_{N+\frac{1}{2}}$ and $y_{\frac{1}{2}}=$ $c<y_{1+\frac{1}{2}} \ldots<d=y_{M+\frac{1}{2}}$. We set $x_{0}=a, x_{i}=\frac{1}{2}\left(x_{i-\frac{1}{2}}+x_{i+\frac{1}{2}}\right)$ for $i=1, \ldots, N, x_{N+1}=b, y_{0}=c$, $y_{j}=\frac{1}{2}\left(y_{j-\frac{1}{2}}+y_{j+\frac{1}{2}}\right)$ for $j=1, \ldots, M, y_{M+1}=d$. We then define a gradient discretisation $D=$ $\left(X_{D, 0}, Y_{D}, \Pi_{D}, \chi_{D}, \nabla_{D}, \operatorname{div}_{D}\right)$ as follows.

1. The space of the discrete velocities $X_{D, 0}$ is defined by

$$
\begin{aligned}
X_{D, 0}= & \left\{u=\left(\left(u_{i+\frac{1}{2}, j}\right)_{i=0, \ldots, N, j=0, \ldots, M+1},\left(u_{i, j+\frac{1}{2}}\right)_{i=0, \ldots, N+1, j=0, \ldots, M}\right):\right. \\
& \left.u_{i+\frac{1}{2}, j} \in \mathbb{R}, u_{i, j+\frac{1}{2}} \in \mathbb{R}, u_{\frac{1}{2}, j}=u_{N+\frac{1}{2}, j}=u_{i, \frac{1}{2}}=u_{i, M+\frac{1}{2}}=0 \text { for all } i, j\right\} .
\end{aligned}
$$

2. The space of the discrete pressures $Y_{D}$ is

$$
Y_{D}=\left\{p=\left(p_{i, j}\right)_{i=1, \ldots, N, j=1, \ldots, M}: p_{i, j} \in \mathbb{R}\right\} .
$$

3. For all $u \in X_{D, 0}, \Pi_{D} u=\left(\Pi_{D}^{(1)} u, \Pi_{D}^{(2)} u\right) \in L^{2}(\Omega)^{2}$ with $\Pi_{D}^{(1)} u$ piecewise constant equal to $u_{i+\frac{1}{2}, j}$ in $\left(x_{i}, x_{i+1}\right) \times\left(y_{j-\frac{1}{2}}, y_{j+\frac{1}{2}}\right)$ for $i=0, \ldots, N$ and $j=1, \ldots, M$, and $\Pi_{D}^{(2)} u$ piecewise constant equal to $u_{i, j+\frac{1}{2}}$ in $\left(x_{i-\frac{1}{2}}, x_{i+\frac{1}{2}}\right) \times\left(y_{j}, y_{j+1}\right)$ for $i=1, \ldots, N$ and $j=0, \ldots, M$ (this definition accounts for the boundary conditions on the velocity).

4. For all $p \in Y_{D, 0}, \chi_{D} p$ is piecewise constant equal to $p_{i, j}$ in $\left(x_{i-\frac{1}{2}}, x_{i+\frac{1}{2}}\right) \times\left(y_{j-\frac{1}{2}}, y_{j+\frac{1}{2}}\right)$ for $i=$ $1, \ldots, N$ and $j=1, \ldots, M$.

5. For all $u \in X_{D, 0}, \nabla_{D} u=\left(\nabla_{D}^{(a, b)} u\right)_{a, b=1,2} \in L^{2}(\Omega)^{4}$ with $\nabla_{D}^{(a, b)} u$ the piecewise constant approximation of the $b$-th derivative of the $a$-th component defined by:

(a) $\nabla_{D}^{(1,1)} u=\frac{u_{i+\frac{1}{2}, j}-u_{i-\frac{1}{2}, j}}{x_{i+\frac{1}{2}}-x_{i-\frac{1}{2}}}$ on $\left(x_{i-\frac{1}{2}}, x_{i+\frac{1}{2}}\right) \times\left(y_{j-\frac{1}{2}}, y_{j+\frac{1}{2}}\right)$ for $i=1, \ldots, N$ and $j=1, \ldots, M$,

(b) $\nabla_{D}^{(1,2)} u=\frac{u_{i+\frac{1}{2}, j+1}-u_{i+\frac{1}{2}, j}}{y_{j+1}-y_{j}}$ on $\left(x_{i}, x_{i+1}\right) \times\left(y_{j}, y_{j+1}\right)$ for $i=0, \ldots, N$ and $j=0, \ldots, M$,

(c) $\nabla_{D}^{(2,1)} u=\frac{u_{i+1, j+\frac{1}{2}}-u_{i, j+\frac{1}{2}}}{x_{i+1}-x_{i}}$ on $\left(x_{i}, x_{i+1}\right) \times\left(y_{j}, y_{j+1}\right)$ for $i=0, \ldots, N$ and $j=0, \ldots, M$,

(d) $\nabla_{D}^{(2,2)} u=\frac{u_{i, j+\frac{1}{2}}-u_{i, j-\frac{1}{2}}}{y_{j+\frac{1}{2}}-y_{j-\frac{1}{2}}}$ on $\left(x_{i-\frac{1}{2}}, x_{i+\frac{1}{2}}\right) \times\left(y_{j-\frac{1}{2}}, y_{j+\frac{1}{2}}\right)$ for $i=1, \ldots, N$ and $j=1, \ldots, M$. 
6. $\operatorname{div}_{D} u=\operatorname{Tr}\left(\nabla_{D} u\right)=\nabla_{D}^{(1,1)} u+\nabla_{D}^{(2,2)} u\left(\right.$ constant in $\left(x_{i-\frac{1}{2}}, x_{i+\frac{1}{2}}\right) \times\left(y_{j-\frac{1}{2}}, y_{j+\frac{1}{2}}\right)$ for $i=1, \ldots, N$ and $j=1, \ldots, M)$.

The gradient scheme (8) stemming from such a gradient discretisation is identical to the standard MAC scheme, classically written using finite differences. Setting $h_{D}=\max _{i, j}\left(x_{i+\frac{1}{2}}-x_{i-\frac{1}{2}}, y_{j+\frac{1}{2}}-y_{j-\frac{1}{2}}\right)$, we have the following result.

Proposition 4.3 (Properties of the MAC gradient discretisation)

For any $m \in \mathbb{N}$ we define a gradient discretisation $D_{m}$ as above, from some finite sequences $\left(x_{i+\frac{1}{2}}\right)_{i=0, \ldots, N_{m}}$ and $\left(y_{j+\frac{1}{2}}\right)_{j=0, \ldots, M_{m}}$ such that $h_{D_{m}}$ tends to 0 as $m \rightarrow \infty$. Then $\left(D_{m}\right)_{m \in \mathbb{N}}$ is coercive, consistent and limit-conforming in the sense of Definitions 2.3, 2.4 and 2.5.

\section{Proof}

We drop the indices $m$ for legibility.

Coercivity: Since the definition of $\nabla_{D}$ corresponds to the discrete gradient of a finite volume scheme on a mesh satisfying the usual orthogonality property, the bound on $C_{D}$ is a consequence of the discrete Poincaré inequality [17, Lemma $9.1 \mathrm{p} 765]$. Note that the control of $\operatorname{div}_{D}$ by $\nabla_{D}$ is straightforward from its definition. The lower bound on $\beta_{D}$ is a consequence of Nečas' result $[27,4]$. Indeed, for any $q \in Y_{D, 0}$ we can find $w \in \boldsymbol{H}_{0}^{1}(\Omega)$ such that $\operatorname{div} w=\chi_{D} q$ and $\|w\|_{\boldsymbol{H}_{0}^{1}(\Omega)} \leq C\left\|_{\chi_{D}} q\right\|_{\boldsymbol{L}^{2}(\Omega)}$. The lower bound on $\beta_{D}$ is obtained by considering $v \in X_{D, 0}$ defined by averaging this function $w$ on all edges, and by applying [17, Lemma 9.4 p 776].

Consistency: The consistency for the pressure stems from the fact that, given a family of meshes whose size tend to 0 , any $L^{2}$ function can be approximated by sequences of piecewise constant functions on the meshes. The consistency for the velocity is equally immediate, since Taylor expansions show that for a regular $\varphi$ the interpolation $u \in X_{D, 0}$ defined by $u_{i+\frac{1}{2}, j}=\varphi\left(x_{i+\frac{1}{2}}, y_{j}\right)$ and $u_{i, j+\frac{1}{2}}=\varphi\left(x_{i}, y_{j+\frac{1}{2}}\right)$ has a reconstruction $\Pi_{D} u$ and a discrete gradient $\nabla_{D} u$ close respectively to $\varphi$ and $\nabla \varphi$ if the mesh size is small.

Limit-CONFORMity: Since in this case the domain is locally star-shaped, we use Proposition 2.6 and Remark 2.7. We start by taking $\varphi \in \mathbf{C}^{\infty}(\bar{\Omega})^{2}$ and we show that $W_{D}(\varphi) \rightarrow 0$ as $h_{D} \rightarrow 0$. The study is simplified by considering each component of the gradient separately. For $u \in X_{D, 0}$ we have

$$
\begin{aligned}
\int_{\Omega} \nabla_{D}^{(1,1)} u \varphi^{(1,1)} \mathrm{d} x \mathrm{~d} y & =\sum_{\substack{i=1, \ldots, N \\
j=1, \ldots, M}} \int_{\left(x_{i-\frac{1}{2}}, x_{i+\frac{1}{2}}\right) \times\left(y_{j-\frac{1}{2}}, y_{j+\frac{1}{2}}\right)} \frac{\left(u_{i+\frac{1}{2}, j}-u_{i-\frac{1}{2}, j}\right) \varphi^{(1,1)}(x, y)}{x_{i+\frac{1}{2}}-x_{i-\frac{1}{2}}} \mathrm{~d} x \mathrm{~d} y \\
& =\sum_{\substack{i=1, \ldots, N \\
j=1, \ldots, M}}\left(u_{i+\frac{1}{2}, j}-u_{i-\frac{1}{2}, j}\right) \varphi_{i, j}\left(y_{j+\frac{1}{2}}-y_{j-\frac{1}{2}}\right)
\end{aligned}
$$

where $\varphi_{i, j}$ is the average of $\varphi^{(1,1)}$ on $\left(x_{i-\frac{1}{2}}, x_{i+\frac{1}{2}}\right) \times\left(y_{j-\frac{1}{2}}, y_{j+\frac{1}{2}}\right)$. Using a discrete integration by parts and the Dirichlet boundary conditions, we may write

$$
\begin{aligned}
\sum_{\substack{i=1, \ldots, N \\
j=1, \ldots, M}}\left(u_{i+\frac{1}{2}, j}-u_{i-\frac{1}{2}, j}\right) \varphi_{i, j}\left(y_{j+\frac{1}{2}}-y_{j-\frac{1}{2}}\right) & =-\sum_{\substack{i=1, \ldots, N \\
j=1, \ldots, M}} u_{i+\frac{1}{2}, j} \frac{\varphi_{i+1, j}-\varphi_{i, j}}{x_{i+\frac{1}{2}}-x_{i-\frac{1}{2}}}\left(y_{j+\frac{1}{2}}-y_{j-\frac{1}{2}}\right)\left(x_{i+\frac{1}{2}}-x_{i-\frac{1}{2}}\right) \\
& =-\int_{\Omega} \Pi_{D}^{(1)} u \partial^{(1)} \varphi^{(1,1)} \mathrm{d} x \mathrm{~d} y+R_{D}
\end{aligned}
$$

where $\left|R_{D}\right| \leq C h_{D}\|u\|_{D}$ with $C$ only depending on $\varphi$ and $C_{D}$ from (3). We used the fact that $\varphi_{i, j}=$ $\varphi^{(1,1)}\left(x_{i}, y_{j}\right)+O\left(\left(x_{i+\frac{1}{2}}-x_{i-\frac{1}{2}}\right)\left(y_{j+\frac{1}{2}}-y_{j-\frac{1}{2}}\right)\right)$, which implies $\frac{\varphi_{i+1, j}-\varphi_{i, j}}{x_{i+\frac{1}{2}}-x_{i-\frac{1}{2}}}=\partial^{(1)} \varphi^{(1,1)}\left(x_{i}, y_{j}\right)+\mathcal{O}\left(y_{j+\frac{1}{2}}-\right.$ $\left.y_{j-\frac{1}{2}}\right)=\partial^{(1)} \varphi^{(1,1)}+\mathcal{O}\left(h_{D}\right)$ on $\left(x_{i}, x_{i+1}\right) \times\left(y_{j-\frac{1}{2}}, y_{j+\frac{1}{2}}\right)$. Combining $(23)$ and $(24)$, we get

$$
\left|\int_{\Omega}\left(\nabla_{D}^{(1,1)} u \varphi^{(1,1)}+\Pi_{D}^{(1)} u \partial^{(1)} \varphi^{(1,1)}\right) \mathrm{d} x \mathrm{~d} y\right| \leq C h_{D}\|u\|_{D} .
$$


Using the same idea for each component of $\nabla_{D}$, we conclude that $W_{D}(\varphi) \rightarrow 0$ as $m \rightarrow \infty$.

We now have to prove that $\widetilde{W}_{D}(\psi) \rightarrow 0$ for all $\psi \in C^{\infty}(\bar{\Omega})$. Since $\operatorname{div}_{D}=\nabla_{D}^{(1,1)}+\nabla_{D}^{(2,2)}$, defining $\varphi \in \mathbf{C}^{\infty}(\bar{\Omega})^{2}$ by $\varphi^{(1,1)}=\varphi^{(2,2)}=\psi$ and $\varphi^{(1,2)}=\varphi^{(2,1)}=0$ we see that $\widetilde{W}_{D}(\psi)=W_{D}(\varphi)$. Since we already established that $W_{D}(\varphi) \rightarrow 0$, this completes the proof.

Remark 4.4 Following the above proof, we see that for $v \in \boldsymbol{H}^{2}(\Omega), q \in H^{1}(\Omega), \varphi \in \boldsymbol{H}^{2}(\Omega)^{d}$ and $\psi \in H^{2}(\Omega)$, the quantities $W_{D}(\varphi), \widetilde{W}_{D}(q), S_{D}(v)$ and $\widetilde{S}_{D}(\psi)$ are all of order $\mathcal{O}\left(h_{D}\right)$. If $(\bar{u}, \bar{p}) \in \boldsymbol{H}^{3}(\Omega) \times$ $H^{1}(\Omega)$, the rates of convergence provided for the MAC method by the theorems in Section 3.1 are therefore of order one (see Remark 3.10).

\subsection{Conforming Taylor-Hood scheme}

We assume here that $\Omega$ is a polyhedral bounded open domain of $\mathbb{R}^{d}, d=2$ or 3 , and we take a simplicial mesh of $\Omega$, that is a mesh $(\mathcal{M}, \mathcal{E}, \mathcal{P})$ in the sense of Definition 4.1 such that for each cell $K$ we have $\operatorname{Card}\left(\mathcal{E}_{K}\right)=d+1$. Each cell is therefore a simplex (triangles in $2 \mathrm{D}$, tetrahedra in $3 \mathrm{D}$ ), and the mesh does not have any hanging node: two neighbouring cells meet along an entire edge/face of each of them. The set of vertices of the mesh, that is the non-empty intersections of the closures of two edges (in 2D) or 3 faces or more (in 3D), is denoted by $\mathcal{V}$. We define $\theta_{\mathcal{M}}=\inf \left\{\frac{h_{K}}{\xi_{K}}: K \in \mathcal{M}\right\}$, where $\xi_{K}$ is the diameter of the largest ball included in $K$.

The Taylor-Hood scheme [30] on such a simplicial mesh can be seen as the gradient scheme corresponding to the gradient discretisation $D=\left(X_{D, 0}, Y_{D}, \Pi_{D}, \chi_{D}, \nabla_{D}, \operatorname{div}_{D}\right)$ defined as follows.

1. The space of the discrete velocities is $X_{D, 0}=\left\{\left(v_{s}\right)_{s \in V^{(2)}}: v_{s} \in \mathbb{R}^{d}\right\}$, where $V^{(2)}=\mathcal{V} \cup\left\{\bar{x}_{\sigma}: \sigma \in\right.$ $\left.\mathcal{E}_{K}\right\}$ is the set of nodes of the $\mathbb{P}^{2}$ finite element discretisation on the simplicial mesh.

2. The space of the discrete pressures is $Y_{D}=\left\{\left(p_{s}\right)_{s \in V^{(1)}}: p_{s} \in \mathbb{R}\right\}$, where $V^{(1)}=\mathcal{V}$ is the set of nodes of the $\mathbb{P}^{1}$ finite element discretisation on the mesh.

3. For all $v \in X_{D, 0}, \Pi_{D} v=\sum_{s \in V^{(2)}} v_{s} \varphi_{s}^{(2)}$, where $\varphi_{s}^{(2)}$ is the scalar $\mathbb{P}^{2}$ finite element basis function associated with the node $s$.

4. For all $p \in Y_{D}, \chi_{D} p=\sum_{s \in V^{(1)}} p_{s} \varphi_{s}^{(1)}$, where $\varphi_{s}^{(1)}$ is the scalar $\mathbb{P}^{1}$ finite element basis function associated with the node $s$.

5. For all $v \in X_{D, 0}, \nabla_{D} v=\nabla\left(\Pi_{D} v\right)$.

6. For all $v \in X_{D, 0}, \operatorname{div}_{D} v=\operatorname{div}\left(\Pi_{D} v\right)$.

\section{Proposition 4.5 (Properties of the Taylor-Hood gradient discretisation)}

Let $\left(\mathcal{M}_{m}, \mathcal{E}_{m}, \mathcal{P}_{m}\right)_{m \in \mathbb{N}}$ be a sequence of simplicial meshes of $\Omega$ such that $\left(\theta_{\mathcal{M}_{m}}\right)_{m \in \mathbb{N}}$ remains bounded, and that $h_{\mathcal{M}_{m}} \rightarrow 0$ as $m \rightarrow \infty$. We assume that every cell of every mesh in the sequence has at least $d$ edges in $\Omega$. Let $D_{m}$ be the gradient discretisation defined as above for $(\mathcal{M}, \mathcal{P}, \mathcal{E})=\left(\mathcal{M}_{m}, \mathcal{P}_{m}, \mathcal{E}_{m}\right)$. Then $\left(D_{m}\right)_{m \in \mathbb{N}}$ is coercive, consistent and limit-conforming in the sense of Definitions 2.3, 2.4 and 2.5.

\section{Proof}

Coercivity: Since $\Pi_{D_{m}}\left(X_{D_{m}, 0}\right)$ is the set of continuous and piecewise $\mathbb{P}^{2}$ functions, it is a subset of $H_{0}^{1}(\Omega)$. The Poincaré inequality $\left\|\Pi_{D_{m}} u\right\|_{L^{2}(\Omega)} \leq \operatorname{diam}(\Omega)\left\|\nabla\left(\Pi_{D_{m}} u\right)\right\|_{L^{2}(\Omega)^{d}}$ is therefore satisfied. Applying [15, Lemma 4.24] to estimate $\beta_{D_{m}}$, we obtain the coercivity of $\left(D_{m}\right)_{m \in \mathbb{N}}$.

Consistency: The consistency is proved in $\left[8\right.$, Theorem 3.1.6] in the general case of $\mathbb{P}^{k}$ finite element, thus we just apply this result with $k=2$ for the discrete velocity and $k=1$ for the discrete pressure.

Limit-CONFORMITY: Because of the definition of $\nabla_{D_{m}}$ and $\operatorname{div}_{D_{m}}, \bar{W}_{D_{m}}, W_{D_{m}}$ and $\left.\widetilde{W}_{D_{m}}\right)$ are identically null. 
Remark 4.6 The consistency in [8, Theorem 3.1.6] gives an $\mathcal{O}\left(h_{\mathcal{M}}^{2}\right)$ estimate on $S_{D}$ for functions in $H^{3}(\Omega)$. Hence, since there is no defect of conformity for the Taylor-Hood method, the rates of convergence provided for this scheme by the theorems in Section 3.1 are therefore of order two (see Remark 3.10).

\subsection{The Crouzeix-Raviart scheme}

As for the Taylor-Hood method, we consider a simplicial mesh $(\mathcal{M}, \mathcal{E}, \mathcal{P})$ of a bounded polyhedral domain $\Omega$. We still set $\theta_{\mathcal{M}}=\inf \left\{\frac{h_{K}}{\xi_{K}}: K \in \mathcal{M}\right\}$, where $\xi_{K}$ is the diameter of the largest ball included in $K$. The Crouzeix-Raviart scheme [9] can be seen as a gradient scheme with the gradient discretisation defined as follows.

1. The space of the discrete velocities is $X_{D, 0}=\left\{v=\left(v_{\sigma}\right)_{\sigma \in \mathcal{E}}: v_{\sigma} \in \mathbb{R}^{d}, v_{\sigma}=0\right.$ for all $\left.\sigma \in \mathcal{E}_{\text {ext }}\right\}$.

2. The space of the discrete pressures is $Y_{D}=\left\{p=\left(p_{K}\right)_{K \in \mathcal{M}}: p_{K} \in \mathbb{R}\right\}$.

3. The linear mapping $\Pi_{D}: X_{D, 0} \rightarrow \boldsymbol{L}^{2}(\Omega)$ is the nonconforming piecewise affine reconstruction of each component of the velocity defined by

$$
\forall v \in X_{D, 0}, \Pi_{D} v=\sum_{\sigma \in \mathcal{E}} v_{\sigma} \varphi_{\sigma}
$$

where $\varphi_{\sigma}$ is the non-conforming $\mathbb{P}^{1}$ basis function associated with the face $\sigma$.

4. The linear mapping $\chi_{D}: Y_{D} \rightarrow L^{2}(\Omega)$ is defined by: for $p \in Y_{D}$ and $K \in \mathcal{M}, \chi_{D} p=p_{K}$ on $K$.

5. The linear mapping $\nabla_{D}: X_{D, 0} \rightarrow \boldsymbol{L}^{2}(\Omega)^{d}$ is the piecewise constant "broken gradient":

$$
\forall v \in X_{D, 0}, \forall K \in \mathcal{M},\left(\nabla_{D} v\right)_{\mid K}=\left(\nabla\left(\Pi_{D} v\right)\right)_{\mid K} .
$$

6. The linear mapping $\operatorname{div}_{D}: X_{D, 0} \rightarrow L^{2}(\Omega)$ is defined by

$$
\forall v \in X_{D, 0}, \forall K \in \mathcal{M}, \operatorname{div}_{D} v=\frac{1}{|K|} \sum_{\sigma \in \mathcal{E}_{K}}|\sigma| v_{\sigma} \cdot \mathbf{n}_{K, \sigma}=\left(\operatorname{div}\left(\Pi_{D} v\right)\right)_{\mid K} .
$$

Remark 4.7 Definition (25) leads to the standard Crouzeix-Raviart scheme. It could be replaced by

$$
\forall v \in X_{D, 0}, \Pi_{D} v=\sum_{\sigma \in \mathcal{E}} v_{\sigma} \cdot \mathbf{n}_{\sigma} \varphi_{\sigma}^{R T}
$$

where $\mathbf{n}_{\sigma}$ is a selected normal vector to the face $\sigma$ and $\varphi_{\sigma}^{R T}$ is the $\boldsymbol{H}_{\mathrm{div}}(\Omega)$ conforming Raviart-Thomas basis function associated with the face $\sigma$ oriented by $\mathbf{n}_{\sigma}$. As mentioned in Remark 3.9, we then recover Property (14).

\section{Proposition 4.8 (Properties of the Crouzeix-Raviart gradient discretisation)}

Let $\left(\mathcal{M}_{m}, \mathcal{E}_{m}, \mathcal{P}_{m}\right)_{m \in \mathbb{N}}$ be a sequence of simplicial meshes of $\Omega$, such that $\left(\theta_{\mathcal{M}_{m}}\right)_{m \in \mathbb{N}}$ remains bounded and $h_{\mathcal{M}_{m}} \rightarrow 0$ as $m \rightarrow \infty$. We define $D_{m}$ as above for $(\mathcal{M}, \mathcal{E}, \mathcal{P})=\left(\mathcal{M}_{m}, \mathcal{E}_{m}, \mathcal{P}_{m}\right)$. Then $\left(D_{m}\right)_{m \in \mathbb{N}}$ is coercive, consistent and limit-conforming in the sense of Definitions 2.3, 2.4 and 2.5.

\section{Proof}

Coercivity: Direct consequence of [15, Lemmas 4.30 and 4.31].

Consistency: The consistency of the operators related to the velocity is shown in [22, Theorem 2.1]. The consistency for the interpolation of the pressure is straightforward since, as $h_{\mathcal{M}_{m}} \rightarrow 0$, any function can be approximated in $L^{2}(\Omega)$ by piecewise constant functions on $\mathcal{M}_{m}$. 
Limit-CONFORMity: Any polyhedral domain is locally star-shaped, we can this use Proposition 2.6 to establish the limit-conformity through $W_{D_{m}}$ and $\widetilde{W}_{D_{m}}$ instead of $\bar{W}_{D_{m}}$. Since smooth functions are dense in $\boldsymbol{H}_{\text {div }}(\Omega)^{d}$ and in $H^{1}(\Omega)$, we only need to study the convergence of $W_{D_{m}}$ and $\widetilde{W}_{D_{m}}$ on smooth functions (see Remark 2.7).

Let us handle $W_{D_{m}}$ first. To simplify the notations, we drop the index $m$. We also only consider one component of the discrete velocity $v \in X_{D, 0}$; we therefore treat $\Pi_{D} v$ as a scalar function and $\nabla_{D} v$ as a function with values in $\mathbb{R}^{d}$. Let $\varphi \in C^{\infty}(\bar{\Omega})$. Since $\nabla_{D} v=\nabla\left(\Pi_{D} v\right)$ on each $K \in \mathcal{M}$, we have

$$
\int_{\Omega}\left(\nabla_{D} v \cdot \varphi+\Pi_{D} v \operatorname{div} \varphi\right) \mathrm{d} x=\sum_{K \in \mathcal{M}_{m}} \int_{K}\left(\nabla\left(\Pi_{D} v\right) \cdot \varphi+\Pi_{D} v \operatorname{div} \varphi\right) \mathrm{d} x=\sum_{\sigma \in \mathcal{E}_{\text {int }}} \int_{\sigma} \varphi \cdot\left[\Pi_{D} v \mathbf{n}\right]_{\sigma} \mathrm{d} \gamma(x)
$$

where $\gamma$ is the $(d-1)$-dimensional measure on $\sigma$, and $\left[\Pi_{D} v \mathbf{n}\right]_{\sigma}=\Pi_{K} v \mathbf{n}_{K, \sigma}+\Pi_{L} v \mathbf{n}_{L, \sigma}$ with $\{K, L\}=\mathcal{M}_{\sigma}$ and $\Pi_{K} v=\left(\Pi_{D} v\right)_{\mid K}$. For any $x \in \sigma$ we have $\Pi_{K} v(x)=\Pi_{K} v\left(\bar{x}_{\sigma}\right)+\nabla_{K} v \cdot\left(x-\bar{x}_{\sigma}\right)$ where $\nabla_{K} v=\left(\nabla_{D} v\right)_{\mid K}$. Since $\Pi_{K} v\left(\bar{x}_{\sigma}\right)=\Pi_{L} v\left(\bar{x}_{\sigma}\right)$ we deduce from (29) that

$$
\int_{\Omega}\left(\nabla_{D} v \cdot \varphi+\Pi_{D} v \operatorname{div} \varphi\right) \mathrm{d} x=\sum_{\sigma \in \mathcal{E}_{\text {int }}} \int_{\sigma} \varphi \cdot\left[\mathbf{n} \otimes \nabla_{D} v\right]_{\sigma}\left(x-\bar{x}_{\sigma}\right) \mathrm{d} \gamma(x) .
$$

The smoothness of $\varphi$ gives, for any $x \in \sigma,\left|\varphi(x)-\varphi\left(\bar{x}_{\sigma}\right)\right| \leq|| \nabla \varphi \|_{\infty} h_{K}$. Moreover, since $\left[\mathbf{n} \otimes \nabla_{D} v\right]_{\sigma}$ is constant over $\sigma$ and $\bar{x}_{\sigma}$ is the centre of gravity of $\sigma$,

$$
\int_{\sigma} \varphi\left(\bar{x}_{\sigma}\right) \cdot\left[\mathbf{n} \otimes \nabla_{D} v\right]_{\sigma}\left(x-\bar{x}_{\sigma}\right) \mathrm{d} \gamma(x)=\varphi\left(\bar{x}_{\sigma}\right) \cdot\left[\mathbf{n} \otimes \nabla_{D} v\right]_{\sigma} \int_{\sigma}\left(x-\bar{x}_{\sigma}\right) \mathrm{d} \gamma(x)=0 .
$$

Introducing $\varphi\left(\bar{x}_{\sigma}\right)$ into (30) and using the bound on $\theta_{\mathcal{M}}$ to write $h_{K}|\sigma| \leq C|K|$ with $C$ not depending on $K \in \mathcal{M}$ or $\sigma \in \mathcal{E}_{K}$, we infer that

$$
\begin{aligned}
\left|\int_{\Omega}\left(\nabla_{D} v \cdot \varphi+\Pi_{D} v \operatorname{div} \varphi\right) \mathrm{d} x\right| & \leq|| \nabla \varphi \|_{\infty} \sum_{\sigma \in \mathcal{E}_{\text {int }}}|\sigma|\left(h_{K}^{2}\left|\nabla_{K} v\right|+h_{L}^{2}\left|\nabla_{L} v\right|\right) \\
& \leq C|| \nabla \varphi \|_{\infty} h_{\mathcal{M}_{m}} \sum_{K \in \mathcal{M}_{m}}\left|\nabla_{K} v\right| \sum_{\sigma \in \mathcal{E}_{K}}|K| \\
& =(d+1) C\|\nabla \varphi\|_{\infty} h_{\mathcal{M}_{m}}|| \nabla_{D} v \|_{L^{1}(\Omega)} .
\end{aligned}
$$

This shows that $W_{D_{m}}(\varphi) \rightarrow 0$ as $m \rightarrow \infty$. The convergence of $\widetilde{W}_{D_{m}}$ is a consequence of the convergence of $W_{D_{m}}$, as in the proof of limit-conformity of the MAC gradient discretisation. If $\psi \in C^{\infty}(\bar{\Omega})$ we let $\varphi \in \mathbf{C}^{\infty}(\bar{\Omega})^{d}$ be defined as the diagonal tensor with $\psi$ in each of its diagonal elements. In each cell $K \in \mathcal{M}$ we have $\operatorname{div}_{D} v=\operatorname{div}\left(\Pi_{D} v\right)=\operatorname{Tr}\left(\nabla\left(\Pi_{D} v\right)\right)=\operatorname{Tr}\left(\nabla_{D} v\right)$ where $\operatorname{Tr}$ is the trace of matrices. A simple inspection thus shows that $\widetilde{W}_{D}(\psi)=W_{D}(\varphi)$. Hence, by the convergence established for $W_{D_{m}}$ we see that $\widetilde{W}_{D_{m}}(\psi) \rightarrow 0$ as $m \rightarrow 0$ and the proof is complete.

Remark 4.9 As in Remark 4.4, following the above proof shows for smooth functions estimates $\mathcal{O}\left(h_{\mathcal{M}}\right)$ on $W_{D}, \widetilde{W}_{D}, S_{D}$ and $\widetilde{S}_{D}$, and the theorems in Section 3.1 thus give order one convergence rates on the Crouzeix-Raviart scheme if $(\bar{u}, \bar{p}) \in \boldsymbol{H}^{3}(\Omega) \times H^{1}(\Omega)$.

\subsection{The HMM extension of the Crouzeix-Raviart scheme}

We now turn to a natural extension of the Crouzeix-Raviart scheme to any polyhedral mesh, namely the "Hybrid Mimetic Mixed" (HMM) scheme [13]. A version of this method was developed and studied for Navier-Stokes equations in [10], but with a less efficient stabilisation and with convergence results less 
detailed than the ones in Section 3. The HMM family of schemes contains the Mimetic Finite Difference method of [5]. We note that these mimetic schemes are different from the ones described in [1,2], where both scalar edge and vector vertex unknowns are used for the velocity. After static condensation to eliminate cell unknowns, the method we present here only uses vector edge unknowns for the velocity and therefore leads to a smaller linear system.

Let $(\mathcal{M}, \mathcal{E}, \mathcal{P})$ be a polyhedral mesh of $\Omega$ in the sense of Definition 4.1. For all $K \in \mathcal{M}$ and $\sigma \in \mathcal{E}_{K}$, we denote by $D_{K, \sigma}$ the cone with vertex $x_{K}$ and basis $\sigma$, that is $D_{K, \sigma}=\left\{t x_{K}+(1-t) y: t \in(0,1), y \in \sigma\right\}$. The HMM gradient discretisation is defined as follows.

1. The space of the discrete velocities is $X_{D, 0}=\left\{v=\left(\left(v_{K}\right)_{K \in \mathcal{M}},\left(v_{\sigma}\right)_{\sigma \in \mathcal{E}}\right): v_{K} \in \mathbb{R}^{d}, v_{\sigma} \in \mathbb{R}^{d}, v_{\sigma}=\right.$ 0 for all $\sigma \in \mathcal{E}_{\text {ext }}$.

2. The space of the discrete pressures is $Y_{D}=\left\{p=\left(p_{K}\right)_{K \in \mathcal{M}}: p_{K} \in \mathbb{R}\right\}$.

3. The linear mapping $\Pi_{D}: X_{D, 0} \rightarrow \boldsymbol{L}^{2}(\Omega)$ is the nonconforming piecewise constant reconstruction in the control volumes of each component of the velocity, defined by

$$
\forall v \in X_{D, 0}, \forall K \in \mathcal{M}, \Pi_{D} v=v_{K} \text { on } K .
$$

4. The linear mapping $\chi_{D}: Y_{D} \rightarrow L^{2}(\Omega)$ is defined, as for the Crouzeix-Raviart gradient discretisation, by

$$
\forall p \in Y_{D}, \forall K \in \mathcal{M}, \chi_{D} p=p_{K} \text { on } K
$$

5. The piecewise constant gradient $\nabla_{D}: X_{D, 0} \rightarrow \boldsymbol{L}^{2}(\Omega)^{d}$ is defined by

$$
\forall K \in \mathcal{M}, \forall \sigma \in \mathcal{E}_{K}, \forall x \in D_{K, \sigma}, \nabla_{D} v(x)=\nabla_{K} v+\frac{\sqrt{d}}{d_{K, \sigma}}\left(\mathcal{A}_{K} \mathcal{R}_{K}\left(V_{K}\right)\right)_{\sigma} \otimes \mathbf{n}_{K, \sigma},
$$

where:

- $\nabla_{K} v=\frac{1}{|K|} \sum_{\sigma \in \mathcal{E}_{K}}|\sigma|\left(v_{\sigma}-v_{K}\right) \otimes \mathbf{n}_{K, \sigma}$,

- $V_{K}=\left(v_{\sigma}-v_{K}\right)_{\sigma \in \mathcal{E}_{K}} \in\left(\mathbb{R}^{d}\right)^{\mathcal{E}_{K}}=\left(\mathbb{R}^{\mathcal{E}_{K}}\right)^{d}$,

- $\mathcal{R}_{K}:\left(\mathbb{R}^{\mathcal{E}_{K}}\right)^{d} \rightarrow\left(\mathbb{R}^{\mathcal{E}_{K}}\right)^{d}$ is defined component-wise by $\mathcal{R}_{K}(\boldsymbol{\xi})^{(i)}=R_{K}\left(\boldsymbol{\xi}^{(i)}\right)$ for $i=1, \ldots, d$, where $R_{K}: \mathbb{R}^{\mathcal{E}_{K}} \rightarrow \mathbb{R}^{\mathcal{E}_{K}}$ is the linear mapping $R_{K}(\xi)=\left(R_{K, \sigma}(\xi)\right)_{\sigma \in \mathcal{E}_{K}}$ with

$$
R_{K, \sigma}(\xi)=\xi_{\sigma}-\left(\frac{1}{|K|} \sum_{\sigma^{\prime} \in \mathcal{E}_{K}}\left|\sigma^{\prime}\right| \xi_{\sigma^{\prime}} \mathbf{n}_{K, \sigma^{\prime}}\right) \cdot\left(\bar{x}_{\sigma}-x_{K}\right) .
$$

- $\mathcal{A}_{K}:\left(\mathbb{R}^{\mathcal{E}_{K}}\right)^{d} \rightarrow\left(\mathbb{R}^{\mathcal{E}_{K}}\right)^{d}$ is defined component-wise for all $i=1, \ldots, d$ by $\mathcal{A}_{K}(\boldsymbol{\xi})^{(i)}=A_{K}^{(i)}\left(\boldsymbol{\xi}^{(i)}\right)$ with $A_{K}^{(i)}$ an isomorphism of the vector space $\operatorname{Im}\left(R_{K}\right) \subset \mathbb{R}^{\mathcal{E}_{K}}$.

6. The linear mapping $\operatorname{div}_{D}: X_{D, 0} \rightarrow L^{2}(\Omega)$ is the discrete divergence operator, defined by

$$
\forall v \in X_{D, 0}, \forall K \in \mathcal{M}, \operatorname{div}_{D} v=\frac{1}{|K|} \sum_{\sigma \in \mathcal{E}_{K}}|\sigma| v_{\sigma} \cdot \mathbf{n}_{K, \sigma}=\frac{1}{|K|} \int_{K} \operatorname{Tr}\left(\nabla_{D} v\right) \mathrm{d} x \text { on } K .
$$

The last equality in (34) is a consequence of [14, Eq. (5.11)] that shows that the average over $K$ of $\nabla_{D} v$ is $\nabla_{K} v$. 
We define $\theta_{D}>0$ as the smallest $\theta>0$ such that

$$
\max \left(\max _{\sigma \in \mathcal{E}_{\text {int }}, K, L \in \mathcal{M}_{\sigma}} \frac{d_{K, \sigma}}{d_{L, \sigma}}, \max _{K \in \mathcal{M}, \sigma \in \mathcal{E}_{K}} \frac{h_{K}}{d_{K, \sigma}}\right) \leq \theta
$$

and

$$
\begin{aligned}
& \forall i=1, \ldots, d, \forall K \in \mathcal{M}, \forall \xi \in \mathbb{R}^{\mathcal{E}_{K}}, \\
& \frac{1}{\theta} \sum_{\sigma \in \mathcal{E}_{K}}\left|D_{K, \sigma}\right|\left|\frac{R_{K, \sigma}(\xi)}{d_{K, \sigma}}\right|^{p} \leq \sum_{\sigma \in \mathcal{E}_{K}}\left|D_{K, \sigma}\right|\left|\frac{\left(A_{K}^{(i)} R_{K}(\xi)\right)_{\sigma}}{d_{K, \sigma}}\right|^{p} \leq \theta \sum_{\sigma \in \mathcal{E}_{K}}\left|D_{K, \sigma}\right|\left|\frac{R_{K, \sigma}(\xi)}{d_{K, \sigma}}\right|^{p} .
\end{aligned}
$$

As noticed in [14, Remark 5.4], the choice $A_{K}^{(i)}=\beta_{K}^{(i)} \operatorname{Id}_{\operatorname{Im}\left(R_{K}\right)}$ for some $\beta_{K}^{(i)} \in\left[\frac{1}{\theta}, \theta\right]$ ensures (36). This choice corresponds to the SUSHI method of [18].

We recall that, when using this gradient discretisation in (8), the values $u_{K}$ can be locally eliminated. This is done by taking in (8) the test function $v \in X_{D, 0}$ such that $v_{K}^{(i)}=1$ for all $i=1, \ldots, d$, and all other degrees of freedom equal to 0 . This enables to compute $u_{K}$ in terms of $\left(u_{\sigma}\right)_{\sigma \in \mathcal{E}_{K}}$. The global resulting system therefore involves only the velocity unknowns at the faces of the mesh. The HMM methods contain the Mimetic Finite Difference schemes which, for triangular meshes and particular choices of $\beta_{K}^{(i)}$, are algebraically identical to the lowest order Raviart-Thomas method [26]. The hybridisation of this method gives the same matrix on the edge unknowns as the Crouzeix-Raviart scheme [6]. Since HMM methods are precisely hybrid schemes (with main unknowns on the edges), we can conclude that HMM is indeed an extension to general polyhedral meshes of the Crouzeix-Raviart scheme.

\section{Proposition 4.10 (Coercivity, consistency and limit-conformity)}

Let $\left(\mathcal{M}_{m}, \mathcal{E}_{m}, \mathcal{P}_{m}\right)_{m \in \mathbb{N}}$ be polyhedral meshes of $\Omega$ in the sense of Definition 4.1 , and let $\left(D_{m}\right)_{m \in \mathbb{N}}$ be the corresponding gradient discretisations, defined as above. We assume that $\left(\theta_{D_{m}}\right)_{m \in \mathbb{N}}$ remains bounded and that $h_{\mathcal{M}_{m}} \rightarrow 0$ as $m \rightarrow \infty$. Then $\left(D_{m}\right)_{m \in \mathbb{N}}$ is coercive, consistent and limit-conforming in the sense of Definitions 2.3, 2.4 and 2.5.

\section{Proof}

Coercivity: The upper bound on $\left(C_{D_{m}}\right)_{m \in \mathbb{N}}$ is an immediate consequence of [14, Lemma 5.3] (to estimate $\Pi_{D_{m}} v$ ) and of the last equality of (34) (to estimate $\operatorname{div}_{D_{m}} v$ ).

A lower bound on $\left(\beta_{D_{m}}\right)_{m \in \mathbb{N}}$ can be obtained as for the MAC and the Crouzeix-Raviart schemes from Nečas' result $[27,4]$. It suffices to interpolate in $X_{D, 0}$ by edge averages a field $w \in \boldsymbol{H}_{0}^{1}(\Omega)$ such that $\operatorname{div} w=\chi_{D} q$ and $\|w\|_{\boldsymbol{H}_{0}^{1}(\Omega)} \leq C\left\|\chi_{D} q\right\|_{L^{2}(\Omega)}$.

Consistency: The consistency for the operators related to the velocity is shown in [14, Lemma 5.5]. The consistency for the interpolation of the pressure is similar to that of the MAC and the Crouzeix-Raviart schemes.

Limit-CONFORMity: Since $\Omega$ is locally star-shaped, we can use Proposition 2.6. The convergence to 0 of $W_{D_{m}}$ is proved in [14, Lemma 5.4]. The convergence of $\widetilde{W}_{D_{m}}$ follows in a similar way as for the MAC and Crouzeix-Raviart schemes, by expressing $\operatorname{div}_{D} v$ in terms of $\operatorname{Tr}\left(\nabla_{D} v\right)$ through (34). If $\psi \in H^{1}(\Omega)$ and $\varphi$ is the $d \times d$ diagonal tensor with $\psi$ on the diagonal, we have $\psi \operatorname{Tr}(T)=\varphi: T$ for any tensor $T$. Hence, denoting by $[f]_{\mathcal{M}}$ the piecewise constant function equal on $K$ to $\frac{1}{|K|} \int_{K} f \mathrm{~d} x$, we have

$$
\int_{\Omega} \psi \operatorname{div}_{D} v \mathrm{~d} x=\int_{\Omega} \psi \operatorname{Tr}\left(\left[\nabla_{D} v\right]_{\mathcal{M}}\right) \mathrm{d} x=\int_{\Omega} \varphi:\left[\nabla_{D} v\right]_{\mathcal{M}} \mathrm{d} x=\int_{\Omega}[\varphi]_{\mathcal{M}}: \nabla_{D} v \mathrm{~d} x .
$$

Since $\nabla \psi=\operatorname{div} \varphi$, this shows that $\widetilde{W}_{D}(\psi) \leq W_{D}(\varphi)+\left\|\varphi-[\varphi]_{\mathcal{M}}\right\|_{L^{2}(\Omega)^{d}}$, and the convergence of $\widetilde{W}_{D}(\psi)$ to 0 follows from the convergence of $W_{D}(\varphi)$ to 0 and the fact that $[\varphi]_{\mathcal{M}} \rightarrow \varphi$ in $\boldsymbol{L}^{2}(\Omega)^{d}$. 
Remark 4.11 Similarly to Remarks 4.4 and 4.9, this proof gives estimates on $W_{D}, \widetilde{W}_{D}, S_{D}$ and $\widetilde{S}_{D}$ that show that the theorems in Section 3.1 provide, for the HMM method, $\mathcal{O}\left(h_{\mathcal{M}}\right)$ error estimates if $(\bar{u}, \bar{p}) \in \boldsymbol{H}^{3}(\Omega) \times H^{1}(\Omega)$.

\section{Proof of the convergence results}

\subsection{Steady problem}

\section{Proof of Theorem 3.3}

Once established, Estimate (9) shows that if the right-hand of the linear system $(8)$ on $\left(u_{D}, p_{D}\right)$ is zero (i.e. if $f=0, G=0$, which implies $\bar{u}=0$ and $\bar{p}=0$ ), then the solution $\left(u_{D}, p_{D}\right)$ is also zero. Hence, this square system is invertible, which ensures the existence and uniqueness of its solution for any right-hand side. We now have to show Estimate (9). Under the hypotheses of the theorem, since $\operatorname{div}(\nabla \bar{u})-\nabla \bar{p}=-f+\operatorname{div}(G)+\eta \bar{u}$ in the distribution sense, we get $\operatorname{div}(\nabla \bar{u}-G)-\nabla \bar{p}=\eta \bar{u}-f \in \boldsymbol{L}^{2}(\Omega)$. Using this relation in $\bar{W}_{D}(\nabla \bar{u}-G, \bar{p})$ we write

$$
\bar{W}_{D}(\nabla \bar{u}-G, \bar{p})=\max _{\substack{v \in X_{D, 0} \\\|v\|_{D}=1}} \int_{\Omega}\left[\nabla_{D} v:(\nabla \bar{u}-G)+\Pi_{D} v \cdot(\eta \bar{u}-f)-\bar{p} \operatorname{div}_{D} v\right] \mathrm{d} x .
$$

Invoking the gradient scheme (8) to replace $\int_{\Omega}-\left(\Pi_{D} v \cdot f+\nabla_{D} v: G\right) \mathrm{d} x$, we can write for any $v \in X_{D, 0}$

$$
\int_{\Omega}\left(\eta\left(\bar{u}-\Pi_{D} u_{D}\right) \cdot \Pi_{D} v+\left(\nabla \bar{u}-\nabla_{D} u_{D}\right): \nabla_{D} v+\left(\chi_{D} p_{D}-\bar{p}\right) \operatorname{div}_{D} v\right) \mathrm{d} x \leq \bar{W}_{D}(\nabla \bar{u}-G, \bar{p})\|v\|_{D} .
$$

Let us define $I_{D}: \boldsymbol{H}_{0}^{1}(\Omega) \rightarrow X_{D, 0}$ and $\widetilde{I}_{D}: L_{0}^{2}(\Omega) \rightarrow Y_{D, 0}$ by

$$
\begin{aligned}
& I_{D} \varphi=\underset{v \in X_{D, 0}}{\operatorname{argmin}}\left(\left\|\Pi_{D} v-\varphi\right\|_{L^{2}(\Omega)}+\left\|\nabla_{D} v-\nabla \varphi\right\|_{L^{2}(\Omega)^{d}}+\left\|\operatorname{div}_{D} v-\operatorname{div} \varphi\right\|_{L^{2}(\Omega)}\right), \\
& \widetilde{I}_{D} \psi=\underset{z \in Y_{D, 0}}{\operatorname{argmin}}\left\|\chi_{D} z-\psi\right\|_{L^{2}(\Omega)} .
\end{aligned}
$$

We also set $\varepsilon_{D}(\bar{u}, \bar{p})=\bar{W}_{D}(\nabla \bar{u}-G, \bar{p})+S_{D}(\bar{u})+\widetilde{S}_{D}(\bar{p})$. From (37) we may write

$$
\begin{aligned}
\int_{\Omega}\left(\eta\left(\Pi_{D} I_{D} \bar{u}-\Pi_{D} u_{D}\right) \cdot \Pi_{D} v\right. & \left.+\left(\nabla_{D} I_{D} \bar{u}-\nabla_{D} u_{D}\right): \nabla_{D} v\right) \mathrm{d} x \\
& +\int_{\Omega}\left(\chi_{D} p_{D}-\chi_{D} \widetilde{I}_{D} \bar{p}\right) \operatorname{div}_{D} v \mathrm{~d} x \leq\left(1+(1+\eta) C_{D}\right) \varepsilon_{D}(\bar{u}, \bar{p})\|v\|_{D} .
\end{aligned}
$$

Thanks to Definition 2.3 we can take $v \in X_{D, 0}$ such that $\|v\|_{D}=1$ and

$$
\int_{\Omega} \chi_{D}\left(p_{D}-\widetilde{I}_{D} \bar{p}\right) \operatorname{div}{ }_{D} v \mathrm{~d} x \geq \beta_{D}\left\|\chi_{D}\left(p_{D}-\widetilde{I}_{D} \bar{p}\right)\right\|_{L^{2}(\Omega)}
$$

Equation (39) then leads to

$$
\left\|\chi_{D}\left(p_{D}-\widetilde{I}_{D} \bar{p}\right)\right\|_{L^{2}(\Omega)} \leq \frac{1+(1+\eta) C_{D}}{\beta_{D}} \varepsilon_{D}(\bar{u}, \bar{p})+\frac{1+\eta C_{D}}{\beta_{D}}\left\|I_{D} \bar{u}-u_{D}\right\|_{D} .
$$

Choosing $v=I_{D} \bar{u}-u_{D}$ in (39) and using $\int_{\Omega} \operatorname{div}_{D} u_{D} \chi_{D} q=0$ for all $q \in Y_{D, 0}$, we can write

$$
\left\|I_{D} \bar{u}-u_{D}\right\|_{D}^{2}+\int_{\Omega} \chi_{D}\left(p_{D}-\widetilde{I}_{D} \bar{p}\right) \operatorname{div}_{D} I_{D} \bar{u} \mathrm{~d} x \leq\left(1+(1+\eta) C_{D}\right) \varepsilon_{D}(\bar{u}, \bar{p})\left\|I_{D} \bar{u}-u_{D}\right\|_{D},
$$


which implies, since $\operatorname{div} \bar{u}=0$,

$$
\left\|I_{D} \bar{u}-u_{D}\right\|_{D}^{2} \leq\left(1+(1+\eta) C_{D}\right) \varepsilon_{D}(\bar{u}, \bar{p})\left\|I_{D} \bar{u}-u_{D}\right\|_{D}+S_{D}(\bar{u})\left\|\chi_{D}\left(p_{D}-\widetilde{I}_{D} \bar{p}\right)\right\|_{L^{2}(\Omega)} .
$$

Thanks to (40) and to the Young inequality $a b \leq \frac{1}{2} a^{2}+\frac{1}{2} b^{2}$, the above estimate yields the existence of $C_{1}$, non-decreasing w.r.t. $1 / \beta_{D}, C_{D}$ and $\eta$, such that $\left\|I_{D} \bar{u}-u_{D}\right\|_{D} \leq C_{1} \varepsilon_{D}(\bar{u}, \bar{p})$. The conclusion follows from the definitions of $I_{D} \bar{u}, \widetilde{I}_{D} \bar{p}$ and $C_{D}$, from the triangle inequality, and from (40).

Proof of Theorem 3.5 The proof follows the same idea as the proof of Theorem 3.3, but considering only functions $v \in E_{D}$. For such functions, owing to (11), Equation (37) can be written

$$
\int_{\Omega}\left(\eta\left(\bar{u}-\Pi_{D} u_{D}\right) \cdot \Pi_{D} v+\left(\nabla \bar{u}-\nabla_{D} u_{D}\right): \nabla_{D} v\right) \mathrm{d} x \leq \bar{W}_{D}(\nabla \bar{u}-G, \bar{p})\|v\|_{D} .
$$

We define $I_{D, E_{D}}: E(\Omega) \rightarrow E_{D}$ by $I_{D, E_{D}} \varphi=\underset{v \in E_{D}}{\operatorname{argmin}}\left(\left\|\Pi_{D} v-\varphi\right\|_{L^{2}(\Omega)}+\left\|\nabla_{D} v-\nabla \varphi\right\|_{L^{2}(\Omega)^{d}}\right)$. From (41) and by definition of $S_{D, E_{D}}$ we deduce

$$
\begin{aligned}
\int_{\Omega}\left(\eta\left(\Pi_{D} I_{D, E_{D}} \bar{u}-\Pi_{D} u_{D}\right) \cdot \Pi_{D} v+\left(\nabla_{D} I_{D, E_{D}} \bar{u}-\right.\right. & \left.\left.\nabla_{D} u_{D}\right): \nabla_{D} v\right) \mathrm{d} x \\
& \leq\left[\left(\eta C_{D}+1\right) S_{D, E_{D}}(\bar{u})+\bar{W}_{D}(\nabla \bar{u}-G, \bar{p})\right]\|v\|_{D} .
\end{aligned}
$$

Choosing $v=I_{D, E_{D}} \bar{u}-u_{D} \in E_{D}$ leads to

$$
\left\|\nabla_{D} I_{D, E_{D}} \bar{u}-\nabla_{D} u_{D}\right\|_{L^{2}(\Omega)^{d}} \leq\left(\eta C_{D}+1\right) S_{D, E_{D}}(\bar{u})+\bar{W}_{D}(\nabla \bar{u}-G, \bar{p})
$$

and the proof of (12) is complete since $\left\|\nabla_{D} I_{D, E_{D}} \bar{u}-\nabla \bar{u}\right\|_{L^{2}(\Omega)^{d}} \leq S_{D, E_{D}}(\bar{u})$. Estimate (13) follows from the definition of $C_{D},(42)$ and $\left\|\Pi_{D} I_{D, E_{D}} \bar{u}-\bar{u}\right\|_{L^{2}(\Omega)^{d}} \leq S_{D, E_{D}}(\bar{u})$.

\section{Proof of Theorem 3.7}

The proof starts from the definition of $W_{D}$ (Proposition 2.6). Since $\nabla \bar{u}-G \in \boldsymbol{H}_{\mathrm{div}}(\Omega)$ and $\operatorname{div}(\nabla \bar{u}-G)=$ $\nabla \bar{p}+\eta \bar{u}-f$ we can write for any $v \in E_{D}$

$$
\int_{\Omega}\left(\nabla_{D} v:(\nabla \bar{u}-G)+\Pi_{D} v \cdot(\nabla \bar{p}+\eta \bar{u}-f)\right) \mathrm{d} x \leq W_{D}(\nabla \bar{u}-G)\|v\|_{D} .
$$

Owing to Assumption (14) and since $v \in E_{D}$, we can remove the term $\nabla \bar{p}$. Using the gradient scheme (8) to replace the terms involving $f$ and $G$, we deduce

$$
\int_{\Omega}\left(\nabla_{D} v:\left(\nabla \bar{u}-\nabla_{D} u\right)+\eta \Pi_{D} v \cdot\left(\bar{u}-\Pi_{D} u\right)\right) \mathrm{d} x \leq W_{D}(\nabla \bar{u}-G)\|v\|_{D} .
$$

Hence, (41) is satisfied with $W_{D}(\nabla \bar{u}-G)$ instead of $\bar{W}_{D}(\nabla \bar{u}-G, \bar{p})$, and the conclusion follows as in the proof of Theorem 3.5.

\subsection{Transient problem}

The existence and uniqueness of the solution of the gradient scheme for the transient Stokes problem is a straightforward consequence of the study of the gradient scheme for the steady problem.

Lemma 5.1 (Existence and uniqueness of the discrete solution) Under Hypothesis (17), let D be a space-time discretisation in the sense of Definition 2.8. Then there exists a unique solution $\left(u_{D}, p_{D}\right)$ to the gradient scheme (20). 
Proof The equation on $\left(u^{(n+1)}, p^{(n+1)}\right)$ in (20) is the gradient scheme (8) for the steady Stokes problem, with $\eta=\delta t^{n+\frac{1}{2}}$ and a right-hand side depending on $u^{(n)}$. Existence and uniqueness of the solution therefore follows from Theorem 3.3.

Let us now establish some a priori estimates on the solution of the scheme.

Lemma 5.2 (Estimates) Under Hypotheses (17), let $D$ be a space-time discretisation in the sense of Definition 2.8 and let $\left(u_{D}, p_{D}\right)$ be the solution of Scheme (20). Then, for all $m=0, \ldots, N$,

$$
\begin{aligned}
\int_{0}^{t^{(m)}} \int_{\Omega}\left|\nabla_{D} u_{D}\right|^{2} \mathrm{~d} x \mathrm{~d} t+\frac{1}{2} \int_{\Omega}\left(\left|\Pi_{D} u_{D}^{(m)}\right|^{2}-\left|\Pi_{D} u_{D}^{(0)}\right|^{2}\right) \mathrm{d} x \leq \\
\\
\int_{0}^{t^{(m)}} \int_{\Omega}\left(f \cdot \Pi_{D} u_{D}+G: \nabla_{D} u_{D}\right) \mathrm{d} x \mathrm{~d} t .
\end{aligned}
$$

Moreover, there exist a universal constant $C_{2} \geq 0$ such that

$$
\begin{aligned}
\left\|\Pi_{D} u_{D}\right\|_{L^{\infty}\left(0, T, \boldsymbol{L}^{2}(\Omega)\right)}+\left\|\nabla_{D} u_{D}\right\|_{\boldsymbol{L}^{2}(\Omega \times(0, T))^{d}} & \\
& \leq C_{2}\left(C_{D}\|f\|_{\boldsymbol{L}^{2}(\Omega \times(0, T))}+\|G\|_{\boldsymbol{L}^{2}(\Omega \times(0, T))^{d}}+\left\|\Pi_{D} u^{(0)}\right\|_{\boldsymbol{L}^{2}(\Omega)}\right) .
\end{aligned}
$$

Proof Putting $v=\delta t^{n+\frac{1}{2}} u_{D}^{(n+1)}$ and $q=p_{D}^{(n+1)}$ in (20) we get

$$
\begin{aligned}
\int_{\Omega}\left(\Pi_{D} u_{D}^{(n+1)}-\Pi_{D} u_{D}^{(n)}\right) \cdot \Pi_{D} u_{D}^{(n+1)} \mathrm{d} x+\int_{t^{(n)}}^{t^{(n+1)}} & \int_{\Omega}\left|\nabla_{D} u_{D}^{(n+1)}\right|^{2} \mathrm{~d} x \mathrm{~d} t= \\
& \int_{t^{(n)}}^{t^{(n+1)}} \int_{\Omega}\left(f \cdot \Pi_{D} u_{D}^{(n+1)}+G: \nabla_{D} u_{D}^{(n+1)}\right) \mathrm{d} x \mathrm{~d} t .
\end{aligned}
$$

Using the inequality $(a-b) \cdot a \geq \frac{1}{2}\left(|a|^{2}-|b|^{2}\right.$ ) (valid for any $a, b \in \mathbb{R}^{d}$ ) on the first term, it comes

$$
\begin{aligned}
\frac{1}{2} \int_{\Omega}\left[\left|\Pi_{D} u_{D}^{(n+1)}\right|^{2}-\left|\Pi_{D} u_{D}^{(n)}\right|^{2}\right] \mathrm{d} x+\int_{t^{(n)}}^{t^{(n+1)}} \int_{\Omega}\left|\nabla_{D} u_{D}^{(n+1)}\right|^{2} \mathrm{~d} x \mathrm{~d} t \leq \\
\\
\int_{t^{(n)}}^{t^{(n+1)}} \int_{\Omega}\left(f \cdot \Pi_{D} u_{D}^{(n+1)}+G: \nabla_{D} u_{D}^{(n+1)}\right) \mathrm{d} x \mathrm{~d} t .
\end{aligned}
$$

The proof of (43) is complete by summing this estimate over $n=0, \ldots, m-1$. Estimate (44) is a straightforward consequence of the definition of $C_{D}$ and Young's inequality applied to (43).

Definition 5.3 (Dual semi-norm on $X_{D, 0}$ ) The semi-norm $|\cdot|_{*, D}$ is defined on $X_{D, 0}$ by

$$
|w|_{*, D}=\sup \left\{\int_{\Omega} \Pi_{D} w \cdot \Pi_{D} v \mathrm{~d} x: v \in E_{D},\|v\|_{D}=1\right\}
$$

where we recall that $E_{D}$ is given by (10).

Lemma 5.4 (Estimates on $\left|\delta_{D} u_{D}\right|_{*, D}$ ) Under Hypotheses (17), let $D$ be a space-time discretisation in the sense of Definition 2.8, and let $\left(u_{D}, p_{D}\right)$ be the solution of Scheme (20). We take $C_{3}$ greater than $C_{D}$ and $\left\|\Pi_{D} u^{(0)}\right\|_{L^{2}(\Omega)}$. Then there exist $C_{4} \geq 0$ only depending on $\Omega, d, C_{3}, f$, and $G$ such that

$$
\int_{0}^{T}\left|\delta_{D} u_{D}\right|_{*, D}^{2} \mathrm{~d} t \leq C_{4}
$$


Proof Taking a generic $v \in E_{D}$ such that $\|v\|_{D}=1$ in Scheme (20) and using the definition of $|\cdot|_{*, D}$ gives an estimate

$$
\left|\delta_{D}^{n+\frac{1}{2}} u_{D}\right|_{*, D} \leq C|| \nabla_{D} u_{D}^{(n+1)} \|_{L^{2}(\Omega)^{d}}+\frac{1}{\delta t^{n+\frac{1}{2}}} \int_{t^{(n)}}^{t^{(n+1)}}\left(C_{D}\|f(t)\|_{L^{2}(\Omega)}+\|G\|_{L^{2}(\Omega)^{d}}\right) \mathrm{d} t .
$$

Squaring this estimate, using Jensen's inequality on the time integral, multiplying by $\delta t^{n+\frac{1}{2}}$ and summing over $n$ gives (45) thanks to (44).

We can now prove the first convergence result of gradient schemes for the transient Stokes problem.

\section{Proof of Theorem 3.13}

Step 1: existence of a weak limit of a subsequence of approximations.

Estimate (44) gives the existence of $\bar{u} \in L^{\infty}\left(0, T ; \boldsymbol{L}^{2}(\Omega)\right)$ and $\zeta \in \boldsymbol{L}^{2}(\Omega \times(0, T))^{d}$ such that, up to a subsequence still indexed by $m, \Pi_{D_{m}} u_{D_{m}} \rightarrow \bar{u}$ weakly-* in $L^{\infty}\left(0, T ; \boldsymbol{L}^{2}(\Omega)\right)$ and $\nabla_{D_{m}} u_{D_{m}} \rightarrow \zeta$ weakly in $\boldsymbol{L}^{2}(\Omega \times(0, T))^{d}$. Let $\varphi \in C^{\infty}(\bar{\Omega})^{d}$ and $\theta \in C_{c}^{\infty}(0, T)$. For all $n=0, \ldots, N-1$ and all $t \in\left(t^{(n)}, t^{(n+1)}\right)$ we have

$$
\int_{\Omega} \nabla_{D_{m}} u_{m}^{(n+1)}: \varphi \theta(t)+\Pi_{D_{m}} u_{m}^{(n+1)} \cdot \operatorname{div} \varphi \theta(t) \mathrm{d} x \leq \bar{W}_{D_{m}}(\varphi, 0) \theta(t)\left\|\nabla_{D_{m}} u_{D_{m}}\right\|_{L^{2}(\Omega)^{d}} .
$$

Integrating this over $t \in\left(t^{(n)}, t^{(n+1)}\right)$, summing on $n=0, \ldots, N-1$ and using (44), we find $C_{5}$ not depending on $m$ such that

$$
\int_{0}^{T} \int_{\Omega} \nabla_{D_{m}} u_{D_{m}}: \varphi \theta+\Pi_{D_{m}} u_{D_{m}} \cdot \operatorname{div} \varphi \theta \mathrm{d} x \mathrm{~d} t \leq C_{5} \bar{W}_{D_{m}}(\varphi, 0) .
$$

We pass to the supremum limit as $m \rightarrow \infty$ thanks to the limit-conformity, and we apply the resulting inequality to $\pm \varphi$. This gives

$$
\int_{0}^{T} \int_{\Omega} \zeta:(\varphi \theta)+\bar{u} \cdot \operatorname{div}(\varphi \theta) \mathrm{d} x \mathrm{~d} t=0
$$

With $\varphi \in C_{c}^{\infty}(\Omega)^{d}$ this relation first shows that $\zeta=\nabla \bar{u}$, and therefore that $\bar{u} \in L^{2}\left(0, T ; \boldsymbol{H}^{1}(\Omega)\right)$. Taking then $\varphi$ which does not vanish on $\partial \Omega$, we also infer that the trace of $\bar{u}$ on $\partial \Omega$ is zero and therefore that $\bar{u} \in L^{2}\left(0, T ; \boldsymbol{H}_{0}^{1}(\Omega)\right)$.

Let us now prove that $\bar{u}$ is divergence-free. From Estimate (44) and the coercivity of the sequence of gradient discretisations, we see that $\left(\operatorname{div}_{D_{m}} u_{D_{m}}\right)_{m \in \mathbb{N}}$ (with the same space-time notations as in (21)) is bounded in $L^{2}(\Omega \times(0, T))$. Taking $\psi \in H^{1}(\Omega)$ with zero mean value and $\theta \in C^{\infty}(0, T)$ we have, for any $n=0, \ldots, N-1$ and any $t \in\left(t^{(n)}, t^{(n+1)}\right)$,

$$
\int_{\Omega} \Pi_{D_{m}} u_{D_{m}}^{(n+1)} \cdot \nabla \psi \theta(t)+\operatorname{div}_{D_{m}} u_{D_{m}}^{(n+1)} \psi \theta(t) \mathrm{d} x \leq \bar{W}_{D_{m}}(0, \psi) \theta(t)\left\|\nabla_{D_{m}} u_{D_{m}}\right\|_{\boldsymbol{L}^{2}(\Omega)^{d}} .
$$

As above, we integrate this over $t \in\left(t^{(n)}, t^{(n+1)}\right)$ and sum over $n$ to find

$$
\int_{0}^{T} \int_{\Omega} \Pi_{D_{m}} u \cdot \nabla \psi \theta+\operatorname{div}_{D_{m}} u_{D_{m}} \psi \theta \mathrm{d} x \mathrm{~d} t \leq C_{6} \bar{W}_{D_{m}}(0, \psi)
$$

with $C_{6}$ not depending on $m$. Using the last equation in the gradient scheme (20), we can introduce $\chi_{D_{m}} \widetilde{I}_{D_{m}} \psi$ in the second term of the left-hand side (where $\widetilde{I}_{D_{m}}$ is defined by (38)) and we get

$$
\int_{0}^{T} \int_{\Omega} \Pi_{D_{m}} u \cdot \nabla \psi \theta \mathrm{d} x \mathrm{~d} t \leq\left\|\operatorname{div}_{D_{m}} u_{D_{m}}\right\|_{L^{2}(\Omega \times(0, T))}|| \chi_{D_{m}} \widetilde{I}_{D_{m}} \psi-\psi\left\|_{L_{0}^{2}(\Omega)}\right\| \theta \|_{L^{2}(0, T)}+C_{6} \bar{W}_{D_{m}}(0, \psi) .
$$


We pass to the supremum limit $m \rightarrow \infty$ thanks to the limit-conformity and consistency of the gradient discretisations, and to the bound on $\left(\operatorname{div}_{D_{m}} u_{D_{m}}\right)_{m \in \mathbb{N}}$ in $L^{2}(\Omega \times(0, T))$. Applying the resulting inequality to $\pm \psi$, we deduce that $\int_{0}^{T} \int_{\Omega} \bar{u} \cdot \nabla \psi \theta \mathrm{d} x \mathrm{~d} t=0$. This relation is true for any $\psi \in H^{1}(\Omega)$ with zero mean value, and hence also for any function in $H^{1}(\Omega)$. The shows that $\operatorname{div} \bar{u}=0$.

Step 2: $\bar{u}$ is the solution of (18).

To simplify notations, we drop the indices $m$. Let $\theta \in C_{c}^{\infty}([0, T))$ and $w \in E(\Omega)$. As $(w, 0)$ is the solution of the incompressible steady Stokes problem (Problem (1)) with $f=\eta w$ and $G=\nabla w$, we can find $w_{D} \in X_{D, 0}$ such that $\int_{\Omega} \chi_{D} q \operatorname{div}_{D} w_{D}=0$ for all $q \in Y_{D, 0}, \Pi_{D} w_{D} \rightarrow w$ in $\boldsymbol{L}^{2}(\Omega)$ and $\nabla_{D} w_{D} \rightarrow \nabla w$ in $\boldsymbol{L}^{2}(\Omega)^{d}$ (Theorem 3.3 and Remark 3.4). We take $v=\delta t^{\left(n+\frac{1}{2}\right)} \theta\left(t^{(n)}\right) w_{D}$ as test function in Scheme (20) and we sum the resulting equation on $n=0, \ldots, N-1$ to get $T_{1}+T_{2}+T_{3}=T_{4}$ with

$$
\begin{aligned}
T_{1} & =\sum_{n=0}^{N-1} \delta t^{n+\frac{1}{2}} \theta\left(t^{(n)}\right) \int_{\Omega} \Pi_{D} \delta^{\left(n+\frac{1}{2}\right)} u_{D} \cdot \Pi_{D} w_{D} \mathrm{~d} x, \\
T_{2} & =\sum_{n=0}^{N-1} \delta t^{n+\frac{1}{2}} \theta\left(t^{(n)}\right) \int_{\Omega} \nabla_{D} u_{D}^{(n+1)}: \nabla_{D} w_{D} \mathrm{~d} x, \\
T_{3} & =-\sum_{n=0}^{N-1} \delta t^{n+\frac{1}{2}} \theta\left(t^{(n)}\right) \int_{\Omega} \chi_{D} p_{D}^{(n+1)} \operatorname{div}_{D} w_{D} \mathrm{~d} x, \\
T_{4} & =\sum_{n=0}^{N-1} \theta\left(t^{(n)}\right) \int_{t^{(n)}}^{t^{(n+1)}} \int_{\Omega}\left(f \cdot \Pi_{D} w_{D}+G: \nabla_{D} w_{D}\right) \mathrm{d} x \mathrm{~d} t .
\end{aligned}
$$

First, we remark that $T_{3}=0$ since $\int_{\Omega} \chi_{D} q \operatorname{div}_{D} w_{D}=0$ for all $q \in Y_{D, 0}$. Using discrete integration by parts and writing $\theta\left(t^{(n+1)}\right)-\theta\left(t^{(n)}\right)=\int_{t^{(n)}}^{t^{(n+1)}} \theta^{\prime}$, we find

$$
T_{1}=-\int_{0}^{T} \int_{\Omega} \theta^{\prime} \Pi_{D} u_{D} \cdot \Pi_{D} w_{D} \mathrm{~d} x \mathrm{~d} t-\theta(0) \int_{\Omega} \Pi_{D} u_{D}^{(0)} \cdot \Pi_{D} w_{D} \mathrm{~d} x .
$$

Recall that $u_{D}^{(0)}=J_{D} u_{\text {ini }}$, so that the space-time consistency (Definition 2.9) gives $\Pi_{D} u_{D}^{(0)} \rightarrow u_{\text {ini }}$ in $\boldsymbol{L}^{2}(\Omega)$ as $m \rightarrow \infty$. Thus, by strong convergence in $\boldsymbol{L}^{2}(\Omega)$ of $\Pi_{D} w_{D}$ to $w$,

$$
T_{1} \rightarrow-\int_{0}^{T} \int_{\Omega} \theta^{\prime} \bar{u} \cdot w \mathrm{~d} x \mathrm{~d} t-\theta(0) \int_{\Omega} \bar{u}_{\text {ini }} \cdot w \mathrm{~d} x .
$$

Using the regularity of $\theta$ and the weak convergence of $\nabla_{D} u_{D}$ to $\nabla \bar{u}$ in $\boldsymbol{L}^{2}(\Omega \times(0, T))^{d}$, it easily comes

$$
T_{2} \rightarrow \int_{0}^{T} \theta \int_{\Omega} \nabla \bar{u}: \nabla w \mathrm{~d} x \mathrm{~d} t \quad \text { and } \quad T_{4} \rightarrow \int_{0}^{T} \theta \int_{\Omega}(f \cdot w+G: \nabla w) \mathrm{d} x \mathrm{~d} t .
$$

Passing to the limit in $T_{1}+T_{2}+T_{3}=T_{4}$ concludes the proof that $\bar{u}$ satisfies (18), and thus also (55).

Step 3: convergence in $L^{\infty}\left(0, T ; \boldsymbol{L}^{2}(\Omega)\right)$.

The proof of the uniform-in-time convergence follows the same idea as in [11]. Using Lemma 5.5 below and the generalised Ascoli-Arzela theorem [11, Theorem 6.2], we see that for any $\varphi \in E(\Omega)$ the sequence $\left(\left\langle\Pi_{D_{m}} u_{D_{m}}(\cdot), \varphi\right\rangle_{L^{2}}\right)_{m \in \mathbb{N}}$ is relatively compact in $L^{\infty}(0, T)$. Since this sequence already converges weakly in $L^{2}(0, T)$ towards $\langle\bar{u}(\cdot), \varphi\rangle_{L^{2}}$ (because $\Pi_{D_{m}} u_{D_{m}} \rightarrow \bar{u}$ weakly in $\boldsymbol{L}^{2}(\Omega \times(0, T))$ ), we deduce that $\left\langle\Pi_{D_{m}} u_{D_{m}}(\cdot), \varphi\right\rangle_{\boldsymbol{L}^{2}} \rightarrow\langle\bar{u}(\cdot), \varphi\rangle_{\boldsymbol{L}^{2}}$ uniformly with respect to $t$ as $m \rightarrow \infty$. 
Let $\psi \in \boldsymbol{L}^{2}(\Omega)$ and $\varphi \in E(\Omega)$. By Estimate (44) we have for any $t \in[0, T]$

$$
\left|\left\langle\Pi_{D_{m}} u_{D_{m}}(t), \psi\right\rangle_{\boldsymbol{L}^{2}}-\langle\bar{u}(t), \psi\rangle_{\boldsymbol{L}^{2}}\right| \leq\left|\left\langle\Pi_{D_{m}} u_{D_{m}}(t), \varphi\right\rangle_{\boldsymbol{L}^{2}}-\langle\bar{u}(t), \varphi\rangle_{\boldsymbol{L}^{2}}\right|+C|| \varphi-\left.\psi\right|_{\boldsymbol{L}^{2}(\Omega)}
$$

where $C$ does not depend on $m, t, \varphi$ or $\psi$. Assuming that $\psi$ can be approximated in $\boldsymbol{L}^{2}(\Omega)$ by functions in $E(\Omega)$ (see [31, Ch. I, Theorem 1.4] for a characterisation of such functions $\psi$ ), the preceding estimate and the uniform-in-time convergence of $\left(\left\langle\Pi_{D_{m}} u_{D_{m}}(\cdot), \varphi\right\rangle_{\boldsymbol{L}^{2}}\right)_{m \in \mathbb{N}}$ show that $\left\langle\Pi_{D_{m}} u_{D_{m}}(\cdot), \psi\right\rangle_{\boldsymbol{L}^{2}} \rightarrow\langle\bar{u}(\cdot), \psi\rangle_{\boldsymbol{L}^{2}}$ uniformly-in-time as $m \rightarrow \infty$. As seen in Proposition 6.2 in the appendix, it is known that for any $T_{0} \in[0, T]$ the function $\bar{u}\left(T_{0}\right)$ can be approximated in $\boldsymbol{L}^{2}(\Omega)$ by functions in $E(\Omega)$; hence, we can apply the preceding result to $\psi=\bar{u}\left(T_{0}\right)$. This allows us to see that for any $\left(s_{m}\right)_{m \in \mathbb{N}}$ converging to $T_{0}$

$$
\left\|\bar{u}\left(T_{0}\right)\right\|_{\boldsymbol{L}^{2}(\Omega)}^{2}=\lim _{m \rightarrow \infty}\left\langle\bar{u}\left(s_{m}\right), \bar{u}\left(T_{0}\right)\right\rangle_{\boldsymbol{L}^{2}}=\lim _{m \rightarrow \infty}\left\langle\Pi_{D_{m}} u_{D_{m}}\left(s_{m}\right), \bar{u}\left(T_{0}\right)\right\rangle_{\mathbf{L}^{2}} .
$$

Here, we used the continuity of $\bar{u}:[0, T] \rightarrow \boldsymbol{L}^{2}(\Omega)$ given by (55). Thus, it comes

$$
\left\|\bar{u}\left(T_{0}\right)\right\|_{\boldsymbol{L}^{2}(\Omega)} \leq \liminf _{m \rightarrow \infty}\left\|\Pi_{D_{m}} u_{D_{m}}\left(s_{m}\right)\right\|_{\boldsymbol{L}^{2}(\Omega)} .
$$

Let $k(m)$ such that $s_{m} \in\left(t^{(k(m)-1)}, t^{(k(m))}\right]$, where $\left(t^{l}\right)_{l}$ are the time steps of the discretisation. Definition (21) gives $\Pi_{D_{m}} u_{D_{m}}\left(s_{m}\right)=\Pi_{D_{m}} u_{D_{m}}^{(k(m))}$. The discrete energy estimate (43) therefore leads to

$$
\begin{aligned}
\frac{1}{2} \int_{\Omega}\left|\Pi_{D_{m}} u_{D_{m}}\left(s_{m}\right)\right|^{2} \mathrm{~d} x \leq \frac{1}{2} \int_{\Omega}\left|\Pi_{D_{m}} u_{D_{m}}^{(0)}\right|^{2} \mathrm{~d} x & -\int_{0}^{t^{(k(m))}} \int_{\Omega}\left|\nabla_{D_{m}} u_{D_{m}}\right|^{2} \mathrm{~d} x \mathrm{~d} t \\
& \leq \int_{0}^{t^{(k(m))}} \int_{\Omega}\left(f \cdot \Pi_{D_{m}} u_{D_{m}}+G: \nabla_{D_{m}} u_{D_{m}}\right) \mathrm{d} x \mathrm{~d} t
\end{aligned}
$$

We notice, by weak convergence in $\boldsymbol{L}^{2}(\Omega \times(0, T))^{d}$ of $\nabla_{D_{m}} u_{D_{m}}$ to $\nabla \bar{u}$ and strong convergence in $\boldsymbol{L}^{2}(\Omega \times$ $(0, T))^{d}$ of $\mathbf{1}_{\left[0, t^{(k(m))}\right]} \nabla \bar{u}$ toward $\mathbf{1}_{[0, T]} \nabla \bar{u}$ (due to $t^{(k(m))} \rightarrow T_{0}$ ), where $\mathbf{1}_{A}$ is the characteristic function of $A$,

$$
\begin{aligned}
\int_{0}^{T_{0}} \int_{\Omega}|\nabla \bar{u}|^{2} \mathrm{~d} x \mathrm{~d} t & =\int_{0}^{T} \int_{\Omega} \mathbf{1}_{\left[0, T_{0}\right]} \nabla \bar{u}: \nabla \bar{u} \mathrm{~d} x \mathrm{~d} t \\
& =\lim _{m \rightarrow \infty} \int_{0}^{T} \int_{\Omega} \mathbf{1}_{\left[0, t^{(k(m))}\right]} \nabla \bar{u}: \nabla_{D_{m}} u_{D_{m}} \mathrm{~d} x \mathrm{~d} t \\
& \leq \liminf _{m \rightarrow \infty}\left(\left\|\mathbf{1}_{\left[0, t^{(k(m))}\right]} \nabla \bar{u}||_{\boldsymbol{L}^{2}(\Omega \times(0, T))^{d}}\right\| \mathbf{1}_{\left[0, t^{(k(m))}\right]} \nabla_{D_{m}} u_{D_{m}} \|_{\boldsymbol{L}^{2}(\Omega \times(0, T))^{d}}\right) \\
& =\left\|\mathbf{1}_{\left[0, T_{0}\right]} \nabla \bar{u}\right\|_{\boldsymbol{L}^{2}(\Omega \times(0, T))^{d}} \liminf _{m \rightarrow \infty}\left\|\mathbf{1}_{\left[0, t^{(k(m))}\right]} \nabla_{D_{m}} u_{D_{m}}\right\|_{\boldsymbol{L}^{2}(\Omega \times(0, T))^{d}} .
\end{aligned}
$$

Hence

$$
\int_{0}^{T_{0}} \int_{\Omega}|\nabla \bar{u}|^{2} \mathrm{~d} x \mathrm{~d} t \leq \liminf _{m \rightarrow \infty} \int_{0}^{t^{(k(m))}}\left|\nabla_{D_{m}} u_{D_{m}}\right|^{2} \mathrm{~d} x \mathrm{~d} t
$$

and we can pass to the limit superior in (48) thanks to the weak convergences of $\Pi_{D_{m}} u_{D_{m}}$ and $\nabla_{D_{m}} u_{D_{m}}$. This gives

$$
\begin{aligned}
\limsup _{m \rightarrow \infty} \frac{1}{2}\left\|\Pi_{D_{m}} u_{D_{m}}\left(s_{m}\right)\right\|_{L^{2}(\Omega)}^{2} & \\
& \leq \frac{1}{2} \int_{\Omega}\left|\bar{u}_{\mathrm{ini}}\right|^{2} \mathrm{~d} x-\int_{0}^{T_{0}} \int_{\Omega}|\nabla \bar{u}|^{2} \mathrm{~d} x \mathrm{~d} t+\int_{0}^{T_{0}} \int_{\Omega}(f \cdot \bar{u}+G: \nabla \bar{u}) \mathrm{d} x \mathrm{~d} t .
\end{aligned}
$$


Plugging $\varphi=\bar{u} \mathbf{1}_{\left[0, T_{0}\right]}$ in Problem (55) and integrating by parts, we obtain the continuous energy estimate

$$
\frac{1}{2}\left\|\bar{u}\left(T_{0}\right)\right\|_{\boldsymbol{L}^{2}(\Omega)}^{2}=\frac{1}{2} \int_{\Omega}\left|\bar{u}_{\mathrm{ini}}\right|^{2} \mathrm{~d} x-\int_{0}^{T_{0}} \int_{\Omega}|\nabla \bar{u}|^{2} \mathrm{~d} x \mathrm{~d} t+\int_{0}^{T_{0}} \int_{\Omega}(f \cdot \bar{u}+G: \nabla \bar{u}) \mathrm{d} x \mathrm{~d} t .
$$

Combined with (49) this leads to $\limsup _{m \rightarrow \infty}\left\|\Pi_{D_{m}} u_{D_{m}}\left(s_{m}\right)\right\|_{\boldsymbol{L}^{2}(\Omega)}^{2} \leq\left\|\bar{u}\left(T_{0}\right)\right\|_{\boldsymbol{L}^{2}(\Omega)}^{2}$. Using (47) we deduce $\lim _{m \rightarrow \infty}\left\|\Pi_{D_{m}} u_{D_{m}}\left(s_{m}\right)\right\|_{L^{2}(\Omega)}^{2}=\left\|\bar{u}\left(T_{0}\right)\right\|_{L^{2}(\Omega)}^{2}$. Together with the convergence (46) this allows us to see that $\left\|\Pi_{D_{m}} u_{D_{m}}\left(s_{m}\right)-\bar{u}\left(T_{0}\right)\right\|_{\boldsymbol{L}^{2}(\Omega)}^{2} \rightarrow 0$ (just develop the square). Since $\bar{u}:[0, T] \rightarrow \boldsymbol{L}^{2}(\Omega)$ is continuous we can apply [11, Lemma 5.1] and we conclude that $\Pi_{D_{m}} u_{D_{m}} \rightarrow \bar{u}$ in $\boldsymbol{L}^{2}(\Omega)$ uniformly-in-time.

Step 4: strong convergence of $\nabla_{D_{m}} u_{D_{m}}$.

We write the discrete energy estimate (43) with $t^{(m)}=T$ and use the convergence in $\boldsymbol{L}^{2}(\Omega)^{d}$ of $\Pi_{D_{m}} u_{D_{m}}(T)$ to $\bar{u}(T)$ to see that

$$
\limsup _{m \rightarrow \infty}\left\|\nabla_{D_{m}} u_{D_{m}}\right\|_{L^{2}(\Omega \times(0, T))^{d}}^{2} \leq-\frac{1}{2} \int_{\Omega}\left(|\bar{u}(T)|^{2}-\left|\bar{u}_{\text {ini }}\right|^{2}\right) \mathrm{d} x+\int_{0}^{T} \int_{\Omega}(f \cdot \bar{u}+G: \nabla \bar{u}) \mathrm{d} x \mathrm{~d} t .
$$

The energy estimate (50) with $T_{0}=T$ then shows that

$$
\limsup _{m \rightarrow \infty}\left\|\nabla_{D_{m}} u_{D_{m}}\right\|_{\boldsymbol{L}^{2}(\Omega \times(0, T))^{d}}^{2} \leq\|\nabla \bar{u}\|_{\boldsymbol{L}^{2}(\Omega \times(0, T))^{d}}^{2} .
$$

This allows us to conclude that the weak convergence of $\nabla_{D_{m}} u_{D_{m}}$ to $\nabla \bar{u}$ in $\boldsymbol{L}^{2}(\Omega \times(0, T))^{d}$ is in fact strong.

The following lemma was the initial key to obtain the uniform-in-time convergence result in the previous proof (Step 3).

Lemma 5.5 Under the assumptions and notations in Theorem 3.13, for all $\varphi \in E(\Omega)$ the sequence of functions $t \rightarrow\left\langle\Pi_{D_{m}} u_{D_{m}}(t), \varphi\right\rangle_{\boldsymbol{L}^{2}}$ satisfies the following quasi-equi-continuity property: there exist $C_{7}$ not depending on $m$, and a sequence of real numbers $\left(\omega_{\varphi, D_{m}}\right)_{m \in \mathbb{N}}$ converging to 0 such that for all $t, s \in[0, T]$

$$
\left|\left\langle\Pi_{D_{m}} u_{D_{m}}(t)-\Pi_{D_{m}} u_{D_{m}}(s), \varphi\right\rangle_{L^{2}}\right| \leq C_{7}|t-s|^{\frac{1}{2}}+\omega_{\varphi, D_{m}} .
$$

Proof We drop the index $m$ as in the proof of Theorem 3.13. Let $\varphi \in E(\Omega)$ and consider the solution $\varphi_{D}$ to the steady gradient scheme (8) with $f=\eta \varphi$ and $G=\nabla \varphi$. Then $\Pi_{D} \varphi \rightarrow \varphi$ in $\boldsymbol{L}^{2}(\Omega)$ and $\nabla_{D} \varphi_{D} \rightarrow \nabla \varphi$ in $\boldsymbol{L}^{2}(\Omega)^{d}$. We write $\Pi_{D} u_{D}(t)-\Pi_{D} u_{D}(s)$ as the sum of the jumps of $\Pi_{D} u$ between $t$ and s. Since $\varphi_{D} \in E_{D}$, the definition 5.3 of $|\cdot|_{*, D}$ gives

$$
\begin{aligned}
\left|\left\langle\Pi_{D} u_{D}(t)-\Pi_{D} u_{D}(s), \Pi_{D} \varphi_{D}\right\rangle_{L^{2}}\right| & =\left|\sum_{\substack{n \text { s.t. } \\
s \leq t^{(n)} \leq t}} \delta t^{\left(n+\frac{1}{2}\right)} \int_{\Omega} \Pi_{D} \delta_{D}^{n+\frac{1}{2}} u_{D} \cdot \Pi_{D} \varphi_{D} \mathrm{~d} x\right| \\
& \leq \sum_{\substack{n \text { s.t. } \\
s \leq t^{(n)} \leq t}} \delta t^{\left(n+\frac{1}{2}\right)}\left|\delta_{D}^{n+\frac{1}{2}} u_{D}\right|_{*, D}\left\|\varphi_{D}\right\|_{D} .
\end{aligned}
$$

Since $\nabla_{D} \varphi_{D} \rightarrow \nabla \varphi$ in $\boldsymbol{L}^{2}(\Omega)^{d},\left\|\varphi_{D}\right\|_{D}$ is bounded and so by the Cauchy-Schwarz inequality we may write

$$
\left|\left\langle\Pi_{D} u_{D}(t)-\Pi_{D} u_{D}(s), \Pi_{D} \varphi_{D}\right\rangle_{L^{2}}\right| \leq C\left(\int_{0}^{T}\left|\delta_{D} u_{D}\right|_{*, D}^{2} \mathrm{~d} t\right)^{\frac{1}{2}}(|t-s|+\delta t)^{\frac{1}{2}}
$$


with $C$ not depending on $D$. Thanks to Lemma 5.4 we infer

$$
\left|\left\langle\Pi_{D} u_{D}(t)-\Pi_{D} u_{D}(s), \Pi_{D} \varphi_{D}\right\rangle_{L^{2}}\right| \leq C(|t-s|+\delta t)^{\frac{1}{2}} .
$$

Using Estimate (44) we then write

$$
\begin{aligned}
\left|\left\langle\Pi_{D} u_{D}(t)-\Pi_{D} u_{D}(s), \varphi\right\rangle_{L^{2}}\right| \leq & \left|\left\langle\Pi_{D} u_{D}(t)-\Pi_{D} u_{D}(s), \varphi-\Pi_{D} \varphi_{D}\right\rangle_{L^{2}}\right| \\
& +\left|\left\langle\Pi_{D} u_{D}(t)-\Pi_{D} u_{D}(s), \Pi_{D} \varphi_{D}\right\rangle_{L^{2}}\right| \\
\leq & C\left\|\varphi-\Pi_{D} \varphi_{D}\right\|_{L^{2}(\Omega)}+C(|t-s|+\delta t)^{\frac{1}{2}} \\
\leq & C_{7}|t-s|^{\frac{1}{2}}+C \delta t^{\frac{1}{2}}+C\left\|\varphi-\Pi_{D} \varphi_{D}\right\|_{L^{2}(\Omega)} .
\end{aligned}
$$

The limit-conformity of the sequence of gradient discretisations ensures that $\omega_{\varphi, D}:=C \delta t^{\frac{1}{2}}+C \| \varphi-$ $\Pi_{D} \varphi_{D} \|_{L^{2}(\Omega)}$ tends to 0 , and the proof is complete.

Let us now turn to the proof of the convergence of the pressure (Theorem 3.15).

Lemma 5.6 (Estimates on discrete time-derivative of velocity) Under the assumptions of Theorem 3.15, let $\left(u_{D}, p_{D}\right)$ be the solution of Scheme (20). Let $C_{8}$ be greater than $C_{D}$ and $\left\|J_{D} u_{\mathrm{ini}}\right\|_{D}$. Then there exists $C_{9} \geq 0$ only depending on $\Omega, d, C_{8}$, and $f$ such that

$$
\left\|\Pi_{D} \delta_{D}^{n+\frac{1}{2}} u_{D}\right\|_{L^{2}(\Omega \times(0, T))} \leq C_{9} .
$$

Proof Put $v=\delta_{D}^{n+\frac{1}{2}} u_{D}$ and $q=p_{D}^{(n+1)}$ in Scheme (20). Since $u_{D}^{(0)}=J_{D} \bar{u}_{\text {ini }} \in E_{D}$ we have $v \in E_{D}$ even if $n=0$ and therefore the term involving $\operatorname{div}_{D} v$ disappears. Recalling that $G=0$ here this leads to

$$
\left\|\Pi_{D} \delta_{D}^{n+\frac{1}{2}} u_{D}\right\|_{\boldsymbol{L}^{2}(\Omega)}^{2}+\int_{\Omega} \nabla_{D} u_{D}^{(n+1)}: \nabla_{D} \delta_{D}^{n+\frac{1}{2}} u_{D} \mathrm{~d} x=\frac{1}{\delta t^{n+\frac{1}{2}}} \int_{t^{(n)}}^{t^{(n+1)}} \int_{\Omega} f \cdot \Pi_{D} \delta_{D}^{n+\frac{1}{2}} u_{D} \mathrm{~d} x \mathrm{~d} t .
$$

On the other hand, using $a:(a-b)=\frac{1}{2}\left(|a|^{2}-|b|^{2}\right)+\frac{1}{2}|a-b|^{2} \geq \frac{1}{2}\left(|a|^{2}-|b|^{2}\right)$ for any tensors $a, b$,

$$
\int_{\Omega} \nabla_{D} u_{D}^{(n+1)}: \nabla_{D} \delta_{D}^{n+\frac{1}{2}} u_{D} \mathrm{~d} x \geq \frac{1}{2 \delta t^{n+\frac{1}{2}}} \int_{\Omega}\left[\left|\nabla_{D} u_{D}^{(n+1)}\right|^{2}-\left|\nabla_{D} u_{D}^{(n)}\right|^{2}\right] \mathrm{d} x
$$

Plugging this into (52) and multiplying by $\delta t^{n+\frac{1}{2}}$, it comes

$$
\int_{t^{(n)}}^{t^{(n+1)}}\left\|\Pi_{D} \delta_{D}^{n+\frac{1}{2}} u_{D}\right\|_{L^{2}(\Omega)}^{2} \mathrm{~d} t+\frac{1}{2} \int_{\Omega}\left[\left|\nabla_{D} u_{D}^{(n+1)}\right|^{2}-\left|\nabla_{D} u_{D}^{(n)}\right|^{2}\right] \mathrm{d} x \leq \int_{t^{(n)}}^{t^{(n+1)}} \int_{\Omega} f \cdot \Pi_{D} \delta_{D}^{n+\frac{1}{2}} u_{D} \mathrm{~d} x \mathrm{~d} t .
$$

We sum on $n=0, \ldots, N-1$ to get

$$
\int_{0}^{T}\left\|\Pi_{D} \delta_{D}^{n+\frac{1}{2}} u_{D}\right\|_{L^{2}(\Omega)}^{2} \mathrm{~d} t \leq \frac{1}{2} \int_{\Omega}\left|\nabla_{D} u_{D}^{(0)}\right|^{2} \mathrm{~d} x+\int_{0}^{T} \int_{\Omega} f \cdot \Pi_{D} \delta_{D}^{n+\frac{1}{2}} u_{D} \mathrm{~d} x \mathrm{~d} t .
$$

The Cauchy-Schwarz' and Young's inequalities conclude the proof.

Lemma 5.7 (Estimates on the discrete pressure) Under the assumptions of Theorem 3.15, we let $\left(u_{D}, p_{D}\right)$ be the solution of Scheme (20). Let $C_{10}$ be greater than $C_{D},\left\|J_{D} u_{\text {ini }}\right\|_{D}$ and $\beta_{D}^{-1}$. Then there exists $C_{11} \geq 0$ only depending on $\Omega, d, C_{10}$, and $f$ such that

$$
\left\|\chi_{D} p_{D}\right\|_{L^{2}(\Omega \times(0, T))} \leq C_{11}
$$


Proof Let $v \in X_{D, 0}$ be such that $\|v\|_{D}=1$ and $\beta_{D}\left\|\chi_{D} p_{D}^{(n+1)}\right\|_{L^{2}(\Omega)} \leq \int_{\Omega} \chi_{D} p_{D}^{(n+1)} \operatorname{div}_{D} v \mathrm{~d} x$ (see the definition of $\beta_{D}$ in Definition 2.3). Using $v$ in Scheme (20) we obtain

$$
\begin{aligned}
& \beta_{D}\left\|\chi_{D} p_{D}^{(n+1)}\right\|_{L^{2}(\Omega)} \\
& \quad \leq\left|\int_{\Omega} \Pi_{D} \delta_{D}^{n+\frac{1}{2}} u_{D} \cdot \Pi_{D} v \mathrm{~d} x+\int_{\Omega} \nabla_{D} u_{D}^{(n+1)}: \nabla_{D} v \mathrm{~d} x-\frac{1}{\delta t^{n+\frac{1}{2}}} \int_{t^{(n)}}^{t^{(n+1)}} \int_{\Omega} f \cdot \Pi_{D} v \mathrm{~d} x \mathrm{~d} t\right| .
\end{aligned}
$$

By the Cauchy-Schwarz inequality and the definition of $C_{D}$ we deduce

$$
\beta_{D}\left\|\chi_{D} p_{D}^{(n+1)}\right\|_{L^{2}(\Omega)} \leq C_{D}\left\|\Pi_{D} \delta_{D}^{n+\frac{1}{2}} u_{D}\right\|_{L^{2}(\Omega)}+\left\|u_{D}^{(n+1)}\right\|_{D}+\frac{1}{\delta t^{n+\frac{1}{2}}} \int_{t^{(n)}}^{t^{(n+1)}} C_{D}\|f\|_{L^{2}(\Omega)} \mathrm{d} t .
$$

We take the square of this estimate, multiply the result by $\delta t^{n+\frac{1}{2}}$ and sum over $n$. Estimate (44) and (51) then show that (53) holds.

\section{Proof of Theorem 3.15}

We first apply Theorem 3.13 to get the strong convergence of $\Pi_{D_{m}} u_{D_{m}}$ to $\bar{u}$ in $\boldsymbol{L}^{2}(\Omega \times(0, T))$ and of $\nabla_{D_{m}} u_{D_{m}}$ to $\nabla \bar{u}$ in $\boldsymbol{L}^{2}(\Omega \times(0, T))^{d}$. We recall that $\bar{u} \in L^{2}(0, T ; E(\Omega))$. Thanks to Estimate (53), we can find a function $\bar{p} \in L^{2}\left(0, T, L_{0}^{2}(\Omega)\right)$ such that up to a subsequence $\chi_{D_{m}} p_{D_{m}}$ converges weakly to $\bar{p}$ in $L^{2}(\Omega \times(0, T))$. We then take $\theta \in C_{c}^{\infty}([0, T)), w \in \boldsymbol{H}_{0}^{1}(\Omega)$ and use $v=\delta t^{\left(n+\frac{1}{2}\right)} \theta\left(t^{(n)}\right) I_{D_{m}} w$ as a test function in Scheme (20), were $I_{D}$ is defined by (38). Since $\Pi_{D_{m}} I_{D_{m}} w \rightarrow w$ in $\boldsymbol{L}^{2}(\Omega), \nabla_{D_{m}} I_{D_{m}} w \rightarrow \nabla w$ in $\boldsymbol{L}^{2}(\Omega)^{d}$ and $\operatorname{div}_{D_{m}} I_{D_{m}} w \rightarrow \operatorname{div}(w)$ in $L^{2}(\Omega)$ as $m \rightarrow \infty$, we can pass to the limit in all terms $T_{1}, \ldots, T_{4}$. Note that $T_{3}$ is no longer equal to 0 , but it converges thanks to the weak convergence of $\chi_{D_{m}} p_{D_{m}}$ to $\bar{p}$. We then see that $(\bar{u}, \bar{p})$ satisfy

$$
\left\{\begin{array}{l}
\bar{u} \in L^{2}(0, T, E(\Omega)), \bar{p} \in L^{2}\left(0, T ; L_{0}^{2}(\Omega)\right), \\
\int_{0}^{T} \int_{\Omega}-\bar{u} \cdot \partial_{t} \varphi \mathrm{d} x \mathrm{~d} t+\int_{\Omega} \bar{u}_{\text {ini }} \cdot \varphi(\cdot, 0) \mathrm{d} x+\int_{0}^{T} \int_{\Omega} \nabla \bar{u}: \nabla \varphi \mathrm{d} x \mathrm{~d} t \\
\quad-\int_{0}^{T} \int_{\Omega} \bar{p} \operatorname{div} \varphi \mathrm{d} x \mathrm{~d} t=\int_{0}^{T} \int_{\Omega} f \cdot \varphi \mathrm{d} x \mathrm{~d} t, \forall \varphi=\theta w \text { with } \theta \in C_{c}^{\infty}([0, T)), w \in \boldsymbol{H}_{0}^{1}(\Omega) .
\end{array}\right.
$$

The density for the $\mathbf{C}^{1}(\bar{\Omega} \times[0, T])$-norm of tensorial functions in $\mathbf{C}_{c}^{\infty}(\Omega \times(0, T))$ ensures that this relation is also satisfied for any $\varphi \in \mathbf{C}_{c}^{\infty}(\Omega \times(0, T))$. Since all the terms in the equality except the first one are continuous w.r.t. $\varphi \in \mathbf{C}_{c}^{\infty}(\Omega \times(0, T))$ for the $L^{2}\left(0, T ; \boldsymbol{H}_{0}^{1}(\Omega)\right)$ norm, this shows that $\partial_{t} u \in L^{2}\left(0, T ; \boldsymbol{H}^{-1}(\Omega)\right)$. Since $\bar{u} \in L^{2}\left(0, T ; \boldsymbol{H}_{0}^{1}(\Omega)\right)$ we deduce that $u \in C\left([0, T] ; \boldsymbol{L}^{2}(\Omega)\right)$. This regularity of $\bar{u}$ then allows to perform an integration by parts in order to see that (54) is equivalent to (19).

\section{Appendix}

\subsection{Proof of Proposition 2.6}

Let us first establish a density result, and then use it to prove Proposition 2.6.

Lemma 6.1 Let $\Omega$ be a locally star-shaped open bounded domain in $\mathbb{R}^{d}$, and let $Z(\Omega)$ as in Definition 2.5. The set $C^{\infty}(\bar{\Omega})^{d} \times C^{\infty}(\bar{\Omega})$ is dense in $Z(\Omega)$ for the topology defined by $\|(\varphi, \psi)\|_{Z(\Omega)}=\|\varphi\|_{L^{2}(\Omega)^{d}}+$ $\|\psi\|_{L^{2}(\Omega)}+\|\operatorname{div} \varphi-\nabla \psi\|_{L^{2}(\Omega)}$

Proof The proof follows the ideas of [31, Ch.1, Theorem 1.1, (iii)]. If $U$ is an open set of $\mathbb{R}^{d}$, we define $L: \boldsymbol{L}^{2}(U)^{d} \times L^{2}(U) \rightarrow \mathcal{D}^{\prime}(U)^{d}$ by $L(f, g)=\operatorname{div} f-\nabla g$. We have $Z(U)=\left\{(f, g) \in \boldsymbol{L}^{2}(U)^{d} \times L^{2}(U):\right.$ $\left.L(f, g) \in \boldsymbol{L}^{2}(U)\right\}$ and $\|(f, g)\|_{Z(U)}=\|f\|_{\boldsymbol{L}^{2}(U)^{d}}+\|g\|_{L^{2}(U)}+\|L(f, g)\|_{\boldsymbol{L}^{2}(U)}$. 
Step 1: Stability of $Z(U)$ by localisation.

Let $\theta \in C^{\infty}(\bar{U})$ and $(f, g) \in Z(U)$. Then $L(\theta f, \theta g)=\operatorname{div}(\theta f)-\nabla(\theta g)=\theta L(f, g)+f \nabla \theta+g \nabla \theta \in \boldsymbol{L}^{2}(U)$ and therefore $(\theta f, \theta g) \in Z(U)$. Here, $f$ is considered here as a matrix and $f \nabla \theta$ is therefore a matrixvector product, whereas $g \nabla \theta$ is a scalar-vector product. We also see from this formula for $L(\theta f, \theta g)$ that $(f, g) \rightarrow(\theta f, \theta g)$ is continuous $Z(U) \rightarrow Z(U)$.

Step 2: Stability of $Z(\cdot)$ by dilatation.

Let $\lambda>1$ and define for $h \in L^{2}(U)$ the function $h_{\lambda} \in L^{2}(\lambda U)$ by $h_{\lambda}(x)=h\left(\frac{x}{\lambda}\right)$. Let $(f, g) \in Z(U)$. We have $L\left(f_{\lambda}, g_{\lambda}\right)=\operatorname{div} f_{\lambda}-\nabla g_{\lambda}=\frac{1}{\lambda} L(f, g)(\dot{\bar{\lambda}}) \in \boldsymbol{L}^{2}(\lambda U)$.

It is also well-known that if $U$ is star-shaped with respect to 0 (which implies $U \subset \lambda U$ for all $\lambda \geq 1$ ), for any $h \in L^{2}(U)$ we have $\left(h_{\lambda}\right)_{\mid U} \rightarrow h$ in $L^{2}(U)$ as $\lambda \rightarrow 1$. Hence, the previous formula for $L\left(f_{\lambda}, g_{\lambda}\right)$ shows that $\left(f_{\left.\lambda\right|_{U}}, g_{\left.\lambda\right|_{U}}\right) \rightarrow(f, g)$ in $Z(U)$ as $\lambda \rightarrow 1$.

Step 3: If $U$ is star-shaped with respect to 0 , approximation of elements in $Z(U)$ by elements in $Z\left(\mathbb{R}^{d}\right)$. Let $\lambda>1$. Since $U$ is star-shaped with respect to 0 we have $U \subset \subset \lambda U$ and therefore we can find $\gamma \in C_{c}^{\infty}(\lambda U)$ such that $\gamma=1$ on a neighborhood of $\bar{U}$. By combining Steps 1 and 2 we see that $\left(\gamma f_{\lambda}, \gamma g_{\lambda}\right) \in Z(\lambda U)$. Since $\gamma$ has a compact support in $\lambda U$, extending these functions to 0 outside $\lambda U$ gives $\left(\gamma f_{\lambda}, \gamma g_{\lambda}\right) \in Z\left(\mathbb{R}^{d}\right)$.

Since $\gamma \equiv 1$ on $U$, Step 1 shows that $L\left(\gamma f_{\lambda}, \gamma g_{\lambda}\right)=L\left(f_{\lambda}, g_{\lambda}\right)$ on $U$. Hence, if $\lambda$ is close to 1 then $\left(\gamma f_{\lambda}, \gamma g_{\lambda}\right)_{\mid U}$ is close to $(f, g)$ in $Z(U)$.

Step 4: Approximation by smooth functions in $Z\left(\mathbb{R}^{d}\right)$.

Let $(f, g) \in Z\left(\mathbb{R}^{d}\right)$ and $\rho \in C_{c}^{\infty}\left(\mathbb{R}^{d}\right)$ be such that $\rho \geq 0$ and $\int_{\mathbb{R}^{d}} \rho \mathrm{d} x=1$. For $n \in \mathbb{N}$, let $\rho_{n}$ denote the function $x \rightarrow n^{d} \rho(n x)$. Then $\left(f * \rho_{n}, g * \rho_{n}\right) \in C^{\infty}\left(\mathbb{R}^{d}\right)^{d} \times C^{\infty}\left(\mathbb{R}^{d}\right)$ and $\left\|f * \rho_{n}-f\right\|_{L^{2}\left(\mathbb{R}^{d}\right)^{d}}+\| g * \rho_{n}-$ $g \|_{\boldsymbol{L}^{2}\left(\mathbb{R}^{d}\right)} \rightarrow 0$ as $n \rightarrow \infty$. Moreover,

$$
L\left(f * \rho_{n}, g * \rho_{n}\right)=\operatorname{div}\left(f * \rho_{n}\right)-\nabla\left(g * \rho_{n}\right)=\operatorname{div}(f) * \rho_{n}-\nabla g * \rho_{n}=L(f, g) * \rho_{n} .
$$

Hence, $L\left(f * \rho_{n}, g * \rho_{n}\right) \rightarrow L(f, g)$ in $\boldsymbol{L}^{2}\left(\mathbb{R}^{d}\right)$ and therefore $\left(f * \rho_{n}, g * \rho_{n}\right) \rightarrow(f, g)$ in $Z\left(\mathbb{R}^{d}\right)$ as $n \rightarrow \infty$.

Step 5: Conclusion.

Now, let $(\varphi, \psi) \in Z(\Omega)$ and $\left(\mathcal{O}_{j}\right)_{j \in J}$ be an open cover of $\bar{\Omega}$ such that for all $j \in J$ the open set $\Omega \cap \mathcal{O}_{j}$ is star-shaped with respect to some point $s_{j}$. Let us consider a partition of unity $\left(\theta_{j}\right)_{j \in J}$ subordinated to this cover: $\theta_{j} \in C_{c}^{\infty}\left(\mathcal{O}_{j}\right)$ and $1=\sum_{j \in J} \theta_{j}$ on $\Omega$. We have $\varphi=\sum_{j \in J} \theta_{j} \varphi$ and $\psi=\sum_{j \in J} \theta_{j} \psi$. Let us define, for $n \in \mathbb{N}$ and $\lambda>1$,

$$
\varphi_{n, \lambda}=\sum_{j \in J}\left(\theta_{j} \varphi\right)_{\lambda} * \rho_{n} \quad \text { and } \quad \psi_{n, \lambda}=\sum_{j \in J}\left(\theta_{j} \psi\right)_{\lambda} * \rho_{n} .
$$

Here, $(\cdot)_{\lambda}$ denotes for each $j \in J$ the dilatation with respect to $s_{j}$. The four previous steps show that $\left(\varphi_{n}, \psi_{n}\right) \rightarrow(\varphi, \psi)$ in $Z(\Omega)$ as $n \rightarrow \infty$, and the proof is complete since $\left(\varphi_{n}, \psi_{n}\right) \in \boldsymbol{C}^{\infty}(\bar{\Omega})^{d} \times C^{\infty}(\bar{\Omega})$.

\section{Proof of Proposition 2.6.}

We assume that $W_{D_{m}}(\varphi) \rightarrow 0$ and $\widetilde{W}_{D_{m}}(\psi) \rightarrow 0$ for any $(\varphi, \psi) \in \boldsymbol{H}_{\text {div }}(\Omega)^{d} \times H^{1}(\Omega)$. Let $(\varphi, \psi) \in Z(\Omega)$ and let us prove that $\bar{W}_{D_{m}}(\varphi, \psi) \rightarrow 0$. We recall the definition of $\bar{W}_{D_{m}}$ :

$$
\bar{W}_{D_{m}}(\varphi, \psi)=\max _{\substack{v \in X_{D_{m}, 0} \\\|v\|_{D_{m}}=1}}\left(\int_{\Omega}\left[\nabla_{D_{m}} v: \varphi+\Pi_{D_{m}} v \cdot(\operatorname{div} \varphi-\nabla \psi)-\psi \operatorname{div}_{D_{m}} v\right] \mathrm{d} x\right) .
$$

Using Lemma 6.1 , we can find $(\bar{\varphi}, \bar{\psi}) \in C^{\infty}(\bar{\Omega})^{d} \times C^{\infty}(\bar{\Omega})$ and $\varepsilon>0$ such that $\|(\varphi-\bar{\varphi}, \psi-\bar{\psi})\|_{Z(\Omega)} \leq \varepsilon$. Thanks to the coercivity of the sequence of gradient discretisations, we may write

$$
\bar{W}_{D_{m}}(\varphi, \psi) \leq \bar{W}_{D_{m}}(\bar{\varphi}, \bar{\psi})+\varepsilon\left(1+2 C_{P}\right) .
$$


Moreover, $\bar{\varphi}$ and $\bar{\psi}$ are regular enough to split $\operatorname{div} \bar{\varphi}-\nabla \bar{\psi}$ in the definition of $\bar{W}_{D_{m}}(\varphi, \psi)$, which gives

$$
\bar{W}_{D_{m}}(\varphi, \psi) \leq W_{D_{m}}(\bar{\varphi})+\widetilde{W}_{D_{m}}(\bar{\psi})+\mathcal{O}(\varepsilon) .
$$

Passing to the supremum limit $m \rightarrow \infty$ and the limit $\varepsilon \rightarrow 0$ shows that $\bar{W}_{D_{m}}(\varphi, \psi) \rightarrow 0$ as $m \rightarrow \infty$. The converse is trivial since for all $\varphi \in \boldsymbol{H}_{\text {div }}(\Omega)$ we have $(\varphi, 0) \in Z(\Omega)$ with $W_{D_{m}}(\varphi, 0)=W_{D_{m}}(\varphi)$, and for all $\psi \in H^{1}(\Omega)$ we have $(0, \psi) \in Z(\Omega)$ with $\bar{W}_{D_{m}}(0, \psi)=\widetilde{W}_{D_{m}}(\psi)$.

\subsection{About the solution of the transient Stokes problem}

The theoretical study of the transient Stokes problem, and in particular of the notion of pressure in this model, is significantly more complex than for the steady problem. The initial sense of weak solution for (2) given by Definition 3.11 only involves the velocity unknown as the pressure has been eliminated by the choice of divergence-free test functions. The interest of this formulation is that it provides an existence and uniqueness result based on classical variational arguments [31, Ch.III, Theorem 1.1].

An equivalent and useful formulation of (18) is the following:

$$
\left\{\begin{array}{l}
\bar{u} \in L^{2}(0, T ; E(\Omega)) \cap C\left([0, T] ; \boldsymbol{L}^{2}(\Omega)\right), \partial_{t} \bar{u} \in L^{2}\left(0, T ; E(\Omega)^{\prime}\right), \\
\int_{0}^{T}\left\langle\partial_{t} \bar{u}, \varphi\right\rangle \mathrm{d} t+\int_{0}^{T} \int_{\Omega} \nabla \bar{u}: \nabla \varphi \mathrm{d} x \mathrm{~d} t \\
\quad=\int_{0}^{T} \int_{\Omega} f \cdot \varphi \mathrm{d} x \mathrm{~d} t+\int_{0}^{T} \int_{\Omega} G: \nabla \varphi \mathrm{d} x \mathrm{~d} t, \quad \forall \varphi \in L^{2}(0, T ; E(\Omega)), \\
\bar{u}(\cdot, 0)=\bar{u}_{\text {ini }} \text { in } L^{2}(\Omega),
\end{array}\right.
$$

where $\langle\cdot, \cdot\rangle$ denotes the duality pairing between $E(\Omega)^{\prime}$ and $E(\Omega)$. See for example the discussion at the end of the proof of Theorem 3.15 on the equivalence between (54) and (19).

A pressure can be recovered in a very weak sense. Proposition 1.1 in [31, Ch.III] establishes the existence of a distribution $\bar{p}$ on $\Omega \times(0, T)$ such that if $\bar{u}$ is the solution of $(55)$ then $(\bar{u}, \bar{p})$ satisfies the PDEs in (2) in the sense of distributions. Additional regularity results on $\bar{p}$ can be obtained if we assume that $u_{\text {ini }} \in E(\Omega)$.

Proposition 6.2 (Regularity result) Let us assume Hypothesis (17), and that $\bar{u}_{\mathrm{ini}} \in E(\Omega)$. We denote by $H$ the closure of $\left\{\varphi \in C_{c}^{\infty}(\Omega)^{d}: \operatorname{div}(\varphi)=0\right\}$ in $\boldsymbol{L}^{2}(\Omega)$. Then the weak solution $(\bar{u}, \bar{p})$ to $(2)$ in the sense of Definition 3.11 (and $\bar{p}$ as in the discussion above) satisfies: $\bar{u} \in L^{2}(0, T, E(\Omega)) \cap C([0, T] ; H)$, $\partial_{t} \bar{u} \in L^{2}\left(0, T, E(\Omega)^{\prime}\right)$ and $\bar{p} \in L^{2}\left(0, T, L_{0}^{2}(\Omega)\right)$.

Proof This is essentially contained in the proof of [31, Ch.III, Theorem 1.1 and Proposition 1.2]. Proposition 1.2 in this reference is proved under more regularity assumption on $\Omega$ and the right-hand side, namely $G$ must not be present and $f$ must be divergence-free. This is actually useful just to recover higher regularity on the solution, that is $H^{2}$ on $\bar{u}$ and $H^{1}$ on $\bar{p}$. Under our assumptions, the proof of [31, Ch.III, Proposition 1.2] gives Proposition 6.2.

These additional regularity results on $(\bar{u}, \bar{p})$ make the proof of Proposition 3.12 obvious. Indeed, testing $\partial_{t} \bar{u}-\Delta \bar{u}+\nabla p=f-\operatorname{div}(G)$ (that is satisfied in the sense of distributions, see above) against $\varphi \in$ $\left.\mathbf{C}_{c}^{\infty}(0, T) \times \Omega\right)$ and using the regularity of $(\bar{u}, \bar{p})$, we see that the equation in (19) holds for any smooth $\varphi$ with compact support. The general case is deduced by density of these functions in $L^{2}\left(0, T ; \boldsymbol{H}_{0}^{1}(\Omega)\right)$.

\section{References}

[1] L. Beirão da Veiga, V. Gyrya, K. Lipnikov, and G. Manzini. Mimetic finite difference method for the Stokes problem on polygonal meshes. J. Comput. Phys., 228(19):7215-7232, 2009. 
[2] L. Beirão da Veiga, K. Lipnikov, and G. Manzini. Error analysis for a mimetic discretization of the steady Stokes problem on polyhedral meshes. SIAM J. Numer. Anal., 48(4):1419-1443, 2010.

[3] M. Bercovier and O. Pironneau. Error estimates for finite element method solution of the Stokes problem in the primitive variables. Numer. Math., 33(2):211-224, 1979.

[4] J. H. Bramble. A proof of the inf-sup condition for the Stokes equations on Lipschitz domains. Math. Models Methods Appl. Sci., 13(3):361-371, 2003. Dedicated to Jim Douglas, Jr. on the occasion of his 75 th birthday.

[5] F. Brezzi, K. Lipnikov, and V. Simoncini. A family of mimetic finite difference methods on polygonal and polyhedral meshes. Math. Models Methods Appl. Sci., 15(10):1533-1551, 2005.

[6] Z. Chen. Equivalence between and multigrid algorithms for mixed and nonconforming methods for second order elliptic problems. East-West J. Numer. Math, 4:1-33, 1994.

[7] E. Chénier, R. Eymard, T. Gallouët, and R. Herbin. An extension of the MAC scheme to locally refined meshes: convergence analysis for the full tensor time-dependent Navier-Stokes equations. Calcolo, 52(1):69-107, 2015.

[8] P. Ciarlet. The finite element method for elliptic problems. North-Holland Publishing Co., Amsterdam-New York-Oxford, 1978. Studies in Mathematics and its Applications, Vol. 4.

[9] M. Crouzeix and P.-A. Raviart. Conforming and nonconforming finite element methods for solving the stationary Stokes equations. I. Rev. Française Automat. Informat. Recherche Opérationnelle Sér. Rouge, 7(R-3):33-75, 1973.

[10] J. Droniou and R. Eymard. Study of the mixed finite volume method for Stokes and Navier-Stokes equations. Numerical methods for partial differential equations, 25(1):137-171, 2009.

[11] J. Droniou and R. Eymard. Uniform-in-time convergence result of numerical method for non-linear parabolic equations. Submitted, 2014.

[12] J. Droniou, R. Eymard, T. Gallouët, C. Guichard, and R. Herbin. Gradient schemes for elliptic and parabolic problems. 2014. In preparation.

[13] J. Droniou, R. Eymard, T. Gallouët, and R. Herbin. A unified approach to mimetic finite difference, hybrid finite volume and mixed finite volume methods. Math. Models Methods Appl. Sci., 20(2):265$295,2010$.

[14] J. Droniou, R. Eymard, T. Gallouët, and R. Herbin. Gradient schemes: a generic framework for the discretisation of linear, nonlinear and nonlocal elliptic and parabolic equations. Math. Models Methods Appl. Sci. (M3AS), 23(13):2395-2432, 2013.

[15] A. Ern and J.-L. Guermond. Theory and practice of finite elements, volume 159. Springer, 2004.

[16] R. Eymard, P. Feron, T. Gallouët, R. Herbin, and C. Guichard. Gradient schemes for the Stefan problem. International Journal On Finite Volumes, 10s, 2013.

[17] R. Eymard, T. Gallouët, and R. Herbin. Finite volume methods. In P. G. Ciarlet and J.-L. Lions, editors, Techniques of Scientific Computing, Part III, Handbook of Numerical Analysis, VII, pages 713-1020. North-Holland, Amsterdam, 2000.

[18] R. Eymard, T. Gallouët, and R. Herbin. Discretization of heterogeneous and anisotropic diffusion problems on general nonconforming meshes SUSHI: a scheme using stabilization and hybrid interfaces. IMA J. Numer. Anal., 30(4):1009-1043, 2010. 
[19] R. Eymard, C. Guichard, and R. Herbin. Small-stencil 3D schemes for diffusive flows in porous media. M2AN, 46:265-290, 2012.

[20] R. Eymard, C. Guichard, R. Herbin, and R. Masson. Gradient schemes for two-phase flow in heterogeneous porous media and Richards equation. ZAMM Z. Angew. Math. Mech., 94(7-8):560585,2014

[21] R. Eymard and R. Herbin. Gradient scheme approximations for diffusion problems. Finite Volumes for Complex Applications VI Problems \& Perspectives, pages 439-447, 2011.

[22] A. Fettah. and T. Gallouët. Numerical approximation of the general compressible Stokes problem. IMA J. Numer. Anal., 33(3):922-951, 2013.

[23] J. Guzmán and M. Neilan. Conforming and divergence-free Stokes elements on general triangular meshes. Math. Comp., 83(285):15-36, 2014.

[24] F. Harlow and J. Welch. Numerical calculation of time-dependent viscous incompressible flow of fluid with a free surface. Physics of Fluids, 8:2182-2189, 1965.

[25] A. Linke. On the role of the Helmholtz decomposition in mixed methods for incompressible flows and a new variational crime. Comput. Methods Appl. Mech. Engrg., 268:782-800, 2014.

[26] R. Liska, M. Shashkov, and V. Ganza. Analysis and optimization of inner products for mimetic finite difference methods on triangular grid. Math. Comput. Simu., 67:55-66, 2004.

[27] J. Nečas. Les méthodes directes en théorie des équations elliptiques. Masson et Cie, Éditeurs, Paris, 1967.

[28] R. Nicolaïdes. Analysis and convergence of the MAC scheme I: The linear problem. SIAM J. Numer. Anal., 29:1579-1591, 1992.

[29] S. Patankar. Numerical heat transfer and fluid flow. volume XIII of Series in Computational Methods in Mechanics and Thermal Sciences. Washington - New York - London: Hemisphere Publishing Corporation; New York. McGraw-Hill Book Company, 1980.

[30] C. Taylor and P. Hood. A numerical solution of the Navier-Stokes equations using the finite element technique. Internat. J. Comput. \& Fluids, 1(1):73-100, 1973.

[31] R. Temam. Navier-Stokes equations, volume 2 of Studies in Mathematics and its Applications. North-Holland Publishing Co., Amsterdam, third edition, 1984. Theory and numerical analysis, With an appendix by F. Thomasset.

[32] R. Verfürth. Numerical solution of mixed finite element problems. In Efficient solutions of elliptic systems (Kiel, 1984), volume 10 of Notes Numer. Fluid Mech., pages 132-144. Vieweg, Braunschweig, 1984.

[33] P. Wesseling. Principles of computational fluid dynamics, volume 29 of Springer Series in Computational Mathematics. Springer-Verlag, Berlin, 2001.

[34] S. Zhang. A new family of stable mixed finite elements for the 3D Stokes equations. Math. Comp., 74(250):543-554, 2005.

[35] S. Zhang. Quadratic divergence-free finite elements on Powell-Sabin tetrahedral grids. Calcolo, 48(3):211-244, 2011. 